\title{
Procera Titanium Milled Framework Surface Preparation Impact on Metal-Resin Implant Fixed Dental Prosthesis, A Clinical Model
}

\author{
William Todd Hegmann \\ West Virginia University
}

Follow this and additional works at: https://researchrepository.wvu.edu/etd

\author{
Recommended Citation \\ Hegmann, William Todd, "Procera Titanium Milled Framework Surface Preparation Impact on Metal-Resin \\ Implant Fixed Dental Prosthesis, A Clinical Model" (2012). Graduate Theses, Dissertations, and Problem \\ Reports. 4864. \\ https://researchrepository.wvu.edu/etd/4864
}

This Thesis is protected by copyright and/or related rights. It has been brought to you by the The Research Repository @ WVU with permission from the rights-holder(s). You are free to use this Thesis in any way that is permitted by the copyright and related rights legislation that applies to your use. For other uses you must obtain permission from the rights-holder(s) directly, unless additional rights are indicated by a Creative Commons license in the record and/ or on the work itself. This Thesis has been accepted for inclusion in WVU Graduate Theses, Dissertations, and Problem Reports collection by an authorized administrator of The Research Repository @ WVU. For more information, please contact researchrepository@mail.wvu.edu. 
Procera Titanium Milled Framework Surface Preparation Impact on Metal-Resin Implant Fixed

Dental Prosthesis, A Clinical Model

William Todd Hegmann, D.D.S.

Thesis/Dissertation submitted to the College of Human Resources and Education at West Virginia University in partial fulfillment of the requirements for the degree of

\section{Masters of Science \\ In Prosthodontics}

Mark Richards D.D.S., M.S., F.A.C.P., Chair Ali Kanawati M.B.A., D.D.S., M.S. Michael Bagby D.D.S., M.S., Ph.D. Mohssen Ghalichebaf D.D.S., M.S.

Division of Graduate Prosthodontics

Morgantown, West Virginia

2012

Keywords: Titanium; Milled; Framework; Surface Preparation; Clinical Model 


\begin{abstract}
Purpose: The purpose of this study is to compare and contrast the difference between airborne-particle abrasion and two common commercially available opaquers during shear testing of clinical models. Comparisons between the type of failure (adhesive, cohesive, or mixed) and surface analysis of the airborne-particle abraded samples will be evaluated.
\end{abstract}

Materials and Methods: 16 identical Nobel Procera titanium alloy frameworks were manufactured to fit a master titanium block fitted with four multi-unit abutments. Metalresin implant fixed dental prosthesis were manufactured to test surface preparations. The variables between framework groups were airborne-particle abrasion and opaquer type in a split sample design. Test groups 1 and 2 and 3 were airborne-particle abraded with aluminum oxide particles sized 100 micron, 250 micron, and Rocatec 30 micron silica modified aluminum oxide (3M ESPE) respectively. Specimens were randomly assigned right and left halves and treated with Ropak UV opaquer (Bredent) and Telio opaquer (Ivoclar Vivadent). Test groups four and five were treated entirely with Telio and Ropak opaquer as described above. The titanium frameworks, however, were divided at the midline and airborne-particle abraded with both $100 \mu$ and $250 \mu$ aluminum oxide particles. All specimens were mounted on a master titanium block fitted with replaceable multi-unit abutments. Specimens were each mounted at a 20-degree tilt to the horizon and placed in a universal testing machine at shear with a crosshead speed of $.5 \mathrm{~mm} / \mathrm{min}$ until failure.

Tested specimens were examined with a surgical loupes (Designs for Vision) at X3.5 magnification for type of failure 1) adhesive, 2) cohesive, 3) mixed and graded as such (A, C, and M).SEM Surface Observation recorded at 250X, 3000X, and 27,000X. Specimens were also examined semi quantitatively with energy dispersive $\mathrm{X}$-ray spectroscopy.

Results A universal testing machine was utilized to test specimens to failure in shear with a crosshead speed of $.5 \mathrm{~mm} / \mathrm{min}$. Results were recorded graphically in Newtons/time with maximum load at failure. Maximum load at failure was recorded in Newtons for each specimen. Oneway analysis of variance (ANOVA) of force by group was performed and is graphically depicted in Chart 2 . No statically significant difference was found among test groups. $100 \mu \mathrm{m}$ air particle abrasion group showed a slightly higher mean than the other abrasion groups. The Telio opaquer group was slightly higher than the Ropak group. This area was observed with X3 magnification and failure type was recorded in Table 1.

Conclusion Within the limits of the study, the following conclusions can be drawn: The air-particle abrasion techniques utilized in this study resulted in no statistically significant difference in terms of load failure. The opaquer techniques utilized in this study resulted in no statically significant difference in terms of load failure. There were no cohesive failures observed in this study. Ropak demonstrated mixed failure when airparticle abraded with $100 \mu \mathrm{m}$ and $250 \mu \mathrm{m}$ aluminum oxide. Telio demonstrated mixed failure when air-particle abraded with Rocatec $30 \mu \mathrm{m}$ silica modified aluminum oxide. 


\section{Introduction}

\section{Background}

The metal-resin implant fixed dental prosthesis, also referred to as a hybrid, has become the standard of care to restore the edentulous patient. When evaluating risk vs. benefit and diagnosis vs. prognosis, the metal-resin implant fixed complete dentures are a viable and cost effective method of treatment. PMMA (polymethylmethacrlyate) also referred to as denture acrylic, is combined with denture teeth to replace any missing anatomical parts for both function and esthetics. Since 1940, PMMA has been commonly utilized as both a removable and a provisional material. (Burns, Beck, \& Nelson, 2003) This material allows repair and replacement with ease, eliminating the need to fabricate a new substructure at the time of replacement. Thus, making a hybrid is an attractive solution with its inexpensive fabrication cost and reparability.

The purpose of this study is to compare and contrast the difference between airborne-particle abrasion and two common commercially available opaquers during shear testing of clinical models. Comparisons between the type of failure (adhesive, cohesive, or mixed) and surface analysis of the airborne-particle abraded samples will be evaluated.

\section{Statement of the Problem}

The fracture of a hybrid causes problems for both the patient and practitioner. Modifying fabrication technique of hybrids may result in a more superior product. This 
paper will examine the effect of increasing the particle size during airborne-particle abrasion to provide increased adherence of the opaquer layer when compared to manufacture recommended techniques. It will also examine if incorporation of silica particles and silane bonding increase adherent properties of the opaquer layer. The goal of this study will be to determine if; one opaquer will outperform the other during model testing, how air-particle abrasion effects the surface of milled titanium alloy, and if these results can be used to modify the current fabrication of the hybrid to make a more durable product.

\section{Significance of the Problem}

Commercially pure titanium and titanium alloys have excellent biocompatibility, good mechanical properties, low density, and a passive oxide layer. (Fujishima, 1995) Bonding polymethylmethacrylate during fabrication of a metal-resin implant fixed dental prosthesis or hybrid is a problem. The thickness layer between titanium substructure and the outside of the prosthesis, as well as the relative undercuts and design, strengthen and reinforce high impact polymethylmethacrylate. These known design enhancements 1) thickness of material and 2) framework support, are in direct opposition to designing implant supported fixed detachable prostheses in vivo with respect to lack of space.

A prosthetic driven plan for esthetics and phonetics, as well as adequate alveloectomies and bone reduction, frequently leave the lab technician fighting to afford space for materials. Inadequate space for materials is prerequisite for early failure of all types of prosthesis. The need for more space and simplicity prompts a search for a 
universal, yet simple design, which can be applied to a myriad of patient situations. Ideal components would include an uncomplicated and structurally sound milled titanium bar with dimension to maintain structural integrity for any implant scenario.

Fingers and projections increase support of the teeth, but present a challenge for fabrication and repair; they also use larger quantities of raw titanium alloy too. There exists a commercial drive to create a substructure which, when applied clinically, could be used for any space scenario encountered. Simplicity would reduce cost and fabrication time along with the added benefit of reparability or replacement utilizing the original framework.

Bonding of PMMA to titanium alloy is poor. During fabrication and in vivo use, the differences in the coefficients of thermal expansion between the materials can result in micro-leakage, staining, and separation of the layers between titanium alloy and PMMA (Bulbul \& Kesim, 2010). Adding an opaquer sandwich layer only complicates matters, allowing an opportunity for additional bonding problems and micro-leakage. Current opaque systems show little bond strength, warranting maximizing the current technique.

Literature review reveals few studies with clinically relevant models. Protocols for the production of titanium alloy reinforced hybrids have been modeled after removable dental prosthesis production. In order to maximize the effectiveness of available materials, experiments of titanium alloy surface treatments and opaquers are needed to increase the longevity of the prosthesis.

\section{Hypothesis}


There is a statically significant difference in the shear bond strength of metal-resin implant fixed dental prosthesis within different sizes of air particle abrasion surface treatments. There is a statistically significant difference in type of failure of a metal-resin implant fixed dental prosthesis (adhesive, cohesive, or mixed) when two commercially available opaquers Telio (Ivoclar) and Ropak (XPdent) are applied.

\section{Definitions}

Adhesion: State in which two dissimilar surfaces are held together by chemical or physical forces or both with or without the aid of an adhesive. Adhesion is one aspect of bonding.

Adhesive: Any substance that joins or creates close adherence of two or more surfaces. Intermediate material that causes two materials to adhere to each other.

Adhesive Failure: Failure occurs at the interface where adhesive comes off cleanly, measures bond strength.

Adhesive Joint: Formed during adhesive bonding often involving one adhesive, two substrates and two interfaces. 
Bond Strength Test: Designed to place the bond in tension or shear stress.

Chemical Adhesion- Bonding at the atomic or molecular level.

Cohesive Failure: Failure occurs within the adhesive material/substrate itself, measures strength of bonding material.

Conversion Prosthesis: Term is applied when retention of a prosthesis is changed from removable to fixed by incorporation of fixtures.

Definitive Prosthesis: Prosthesis to be used over an extended period of time.

Dental Implant: A device specially designed to be placed surgically within or onthe mandibular or maxillary bone as a means of providing for dental replacement. 
Dental Prosthesis: Any device or appliance replacing one or more missing teeth and/or, if required, associated structures. (This is a broad term which includes abutment crowns and abutment inlays/onlays, bridges, dentures, obturators, gingival prostheses.)

Early Loading: Functional loading no earlier than 48 hours after implant placement and no later than 3 months afterward.

Fixed Prosthesis: Non-removable dental prosthesis which is solidly attached to abutment teeth, roots or implants.

Fixed-Removable Prosthesis: Combined prosthesis, one or more parts of which are fixed, and the other(s) attached by devices which allow their detachment, removal and reinsertion by the dentist only.

Framework: the skeletal portion of prosthesis (usually metal, sometimes ceramic) around which and to which are attached the remaining portions of the prosthesis to produce a finished restoration.

Gold Cylinder: A machined implant abutment used to wax and cast metal frameworks. 
Hybrid Prosthesis: A nonspecific term applied to any prosthesis that does not follow conventional design.

Hydrolysis: The chemical breakdown of a compound due to reaction with water.

Implant: Material inserted or grafted into tissue.

Interim Prosthesis: A provisional prosthesis designed for use over a limited period of time, after which it is to be replaced by a more definitive restoration.

Mechanical Adhesion: Retention by the interlocking or the penetration of one phase into the surface of the other

Metal-Resin Implant Fixed Dental Prosthesis: A resin prosthesis reinforced with metal supported by implant fixtures.

Mixed Failure: Failure has qualities of adhesion and cohesion. The adhesive remains 
on the surface of both substrates

One Stage Surgical Protocol/Immediate Loading: A non-submerged, one-stage surgery, which loads the implant within 48 hours of placement.

Opaque: Something that does not transmit light, is not transparent, or translucent.

Opaquer: In prosthesis construction, is a layer that is applied to obscure and prevent transmission of light

Prosthesis: Artificial replacement of any part of the body.

Removable Prosthesis: Complete or partial prosthesis, which after an initial fitting by a dentist, can be removed and reinserted by the patient.

Two Stage Surgical Protocol/Conventional Loading: Obtaining and maintaining soft tissue coverage for 3 - 6 months. Maintaining a non-loaded implant environment for 3 - 6 months. 
Shear: Stress occurs when parts of an object slide by one another.

Shear Strength: The maximum stress that material can withstand before failure in a shear mode of loading.

Stress: Failure Load (N) / Surface Area (mm2)

Thermal Cycling: The process of cycling through two temperature extremes

\section{Assumptions}

1. Milled titanium alloy frameworks are identical.

2. Identical milled titanium frameworks fit passively to the master test block.

3. Thickness and form of processed polymethylmethacrylate is uniform across all specimens with regard to internal framework position.

\section{Limitations}

1. Variation of even application of air-particle abrasion of framework surface.

2. Variation of even brush application of respective primers 
3. Variation in thickness of brush applied opaque layer.

4. Cross contamination of air-particle abrasion, primer application, and opaquer application of split mouth specimens at midline.

5. Variation of surface detail as specimens were randomly invested and flasked from a polyether master model

6. Human error during fabrication of the test samples

\section{Delimitations}

1. The same technician fabricated all samples.

2. The same technician performed all testing.

3. Materials in this study from new, un-opened containers.

4. Split designs were randomly assigned right or left.

5. Prepared frameworks were randomly assigned flasks

6. Specimens were prepared in similar environmental conditions. 


\section{Literature Review}

\section{Historical Development}

Many methods have been utilized throughout history to immediately load implant supported fixed dental prosthesis since Osseo integration in 1982. (Parel, 2011) These techniques have been modified consistently in regards to temporization, second stage loading, and immediate loading. The drive has been to provide patients with a prosthesis on the day of surgery and deliver a final restoration shortly after tissue healing. Non-stop service has been a challenge that has become a reality with the improvement of implant surface technology and more importantly, primary stability.

Original protocol called for implant surgery with an undisturbed healing period of 3 to 6 months. A second surgery was later performed to expose and functionally load the implants. The earliest attempts at immediate loading were in 1965 and utilized cobalt-chromium (Co-Cr) frameworks. (Engstrand, Nannmark, Martensson, Galeus, \& Branemark, 2001) Co-Cr cast frameworks were fabricated on master casts to reinforce PMMA. This provided rigid fixation of the fixtures and a passive fit. (Burns, Beck, \& Nelson, 2003)

During the 1970s, gold alloy castings were used as framework support, but the errors in casting led to the use of luting cements to retain the prosthesis. This 
solved some problems of fit but, it led to retrieve ability problems. (Engstrand, Nannmark, Martensson, Galeus, \& Branemark, 2001) Some practitioners utilized temporary cements, challenging the concept of rigid fixation. Later, manufactures produced machined gold cylinders. This improved fit of the casted precious alloys and allowed screw access retrieve ability. These gold cylinders were used in a variety of methods; to solder, cast, and lute frameworks for support. Schnitmann used gold cylinders to convert complete dentures with auto-polymerizing PMMA on the day of surgery. Balshi and Wolfinger coined the term "Conversion Prosthesis". They utilized the more inexpensive impression copings and PMMA to convert dentures into fixed prosthesis at second stage surgery. (Burns, Beck, \& Nelson, 2003) With machined parts increasing the precision of fit, the standardization of the framework and casting with semiprecious alloys began to increase strength and reduce cost.

With rising cost of precious and semi-precious alloys, attempts to reinforce PMMA with carbon and graphite fiber have been utilized with some success. Jemt and coworkers investigated titanium as a framework material. Titanium is difficult to cast with precision and is often non-uniform in properties. Accurate casting of titanium is an expensive undertaking and must be executed in a vacuum environment with special investments. (Craig \& Powers, ) Recent developments in CAD/CAM techniques, allow the manufactures to produce a low cost, biocompatible, custom, precision fit framework to rigidly fixate implants for restoration. 
The type of treatment available today has been driven by patient demand, cost of materials, and the clinicians desire to provide same day service. Utilizing the Branemark Novum technique, in May of 1997, a patient received a metal resin implant fixed prosthesis on the day of implant surgery. (Engstrand, Nannmark, Martensson, Galeus, \& Branemark, 2001)

\section{Metal-Resin Implant Fixed Dental Prosthesis}

Metal-resin implant fixed dental prosthesis have a long clinical tract record despite the limited time line at which they were loaded. Research shows high success rates of immediate loaded dental implants, offering our patients timely solutions. Many authors have commented on design ideas and techniques to increase success of the prosthesis. The number of implants needed to support full arch restorations is quite controversial in the literature. Numbers range from four to as many as can fit. More implants distribute forces and can be useful in patients with larger prosthesis or increased forces. Fewer implants allow for easy cleaning, maintenance, and decreased cost of fabrication.

Parel developed a risk assessment protocols when treating patients with four implants, taking into consideration facts such as; maxillary prosthesis experience implant failure five to six times more than mandibular prosthesis, and that men are three times more likely to experience implant failure than women. Parel termed high-risk patients to include men, patients with poor bone density, and patients 
with opposing natural dentition. Some low risk factors included systemic factors, local infections, opposing implant supported fixed dental prosthesis, bone volume, smoking, bruxism, and distal posterior implant site. (Parel, 2011) It is the opinion of this author that when increased stresses are apparent, more implant support should be incorporated into the prosthesis. Shackleton concluded that when prosthesis have cantilever lengths of less than $15 \mathrm{~mm}$ the survived significantly better than those greater than $15 \mathrm{~mm}$. (Shackleton, 1994)

If designed properly, the most common complication of metal-resin implant fixed dental prosthesis is fracture of the PMMA. (Segerstrã $\llbracket \mathrm{m} \&$ Ruyter, 2009) According to Ohkubo, machined titanium resin implant fixed dental prosthesis have demonstrated bonding problems. (Ohkubo, Watanabe, Hosoi, \& Okabe, 2000) Fractures of the PMMA and bonding problems will become more apparent as an increasing number of metal-resin implant fixed dental prosthesis are employed.

\section{CAD/CAM Titanium Alloy Frameworks}

Computer numeric controlled milling ( $\mathrm{CNC}$ ) has given us the opportunity to create frameworks out of virtually any metal. The ideal framework according to Bulbul and Kesim should be nontoxic, non-allergenic, corrosion resistant, easy to use, relatively inexpensive, and have adequate strength. (Bulbul \& Kesim, 2010) In order for the framework to impart stability and rigidity its fit should be passive imparting no stress upon the implant fixtures as fixation screws are torqued. 
Multiple studies in the literature have demonstrated that CNC-milled titanium frameworks have not only passive but more accurate fit than casted gold alloy frameworks. In an early study, Engstrand and colleges tested multiple frameworks on the same master cast and found that the range of distortion between CNC-milled titanium frameworks and conventional cast gold alloy is similar. He also noted that in his model extensive recontouring of the titanium frameworks in the laboratory was often necessary and time consuming. (Engstrand, Nannmark, Martensson, Galeus, \& Branemark, 2001) This early study did not incorporate the ability of the new technologies to incorporate the design of the final prosthesis that has all but eliminated the need for laboratory recontouring. Ortorp conducted fit testing of CNC-milled titanium frameworks and conventional castings before and after veneering of porcelain for fixed prosthesis. The CNC frameworks showed a statistically better fit and precision of fabrication compared to conventional castings $(\mathrm{P}<.05)$. Application of veneering porcelain did not affect the titanium frameworks statistically $(\mathrm{P}<.05)$. This provided evidence that fabrication of implant supported titanium milled frameworks could be done with precision and repeatability. (Ortorp, Jemt, Back, \& Jalevik, 2003) Al-Fadda et al conducted in vitro studies of CNC-milled titanium and semi-precious metal casting. Within the limits of this study he concluded that the CNC-milling technique yields a statistically significant more accurate fit than the cast technique but in vivo studies are warranted. (Al-Fadda, Zarb, \& Finer, 2007)

Colleges followed up this study with an in vivo study of 126 patients receiving prosthetic rehabilitation. The test group received $67 \mathrm{CNC}$-milled titanium 
frameworks and the control group received 63 conventional prosthesis casted with gold cylinders. No fractures of the titanium frameworks were noted, however two frameworks fractured in the conventional cast group and one framework screw fracture. After three years it was conclude that the CNC-milled titanium frameworks preformed similar clinically and can be a viable alternative to the conventional cast technique. (- Örtorp \& - Jemt, a) They continued their work with this study for two additional years. During this time no fractures of the CNC-milled titanium frameworks were noted, however, both test groups exhibited fractures of the PMMA resin. The performance of both frameworks was similar, both clinically and radiographically, and the $\mathrm{CNC}$-milled frameworks had fewer complications prosthetically. (- Örtorp \& - Jemt, b)

The resin-metal implant fixed dental prosthesis has been noted in the literature to exhibit PMMA resin veneer fractures after short terms of service. Noted as early as 2000 there are few studies that have tested adhesion of PMMA to titanium alloys. (Ohkubo, Watanabe, Hosoi, \& Okabe, 2000) One author remarked the most common complication of implant-retained prosthesis with the metallic framework is fracture of the PMMA. (Segerstrã $\rrbracket[m$ \& Ruyter, 2009) These fractures are directly related to adequate thickness of the PMMA veneering material among other problems.

\section{Adhesive Joint}


Adhesion or bonding is the process of forming an adhesive joint. The initial substrate is called the adherend, whereas the material producing the interface is generally called the adhesive. If two substrates are being joined, the adhesive produces two interfaces as part of the adhesive joint. (Craig \& Powers, )

According to Restorative Dental Materials, formation of an optimally bonded interface requires the following: (1) the surface of the substrate be clean; (2) the adhesive wet the substrate well, have a low contact angle, and spread onto the surface; (3) adaptations to the substrate produce intimate approximation of the materials without entrapped air or other intervening materials; (4) the interface include the sufficient physical, chemical and/or mechanical strength to resist intraoral forces of debonding; and (5) the adhesive be well cured under the conditions recommended for use. (Craig \& Powers, )

Chemical, physical, and mechanical characteristics of the substrate/adherend and adhesive determine the properties of the adhesive joint. The quality of an adhesive joint depends on resistance to failure. Progression of failure depends on adhesive joint properties, bond environment, and time. (Craig \& Powers, )

Defects on the interface lead to crack formation, propagation, and cause interfacial debonding of adhesive joints resulting in joint failure. (Gladwin \& Bagby, 2009) According to Restorative Dental Materials, defects include the following: interfacial contamination, excess moisture, trapped air bubbles, voids formed during solvent evaporation, poor wetting, bubbles within the adhesive, and curing shrinkage pores. (Craig \& Powers, ) 
The adhesive joint is dependant on the chemical bonding of PMMA to the titanium alloy of metal-resin implant fixed dental prosthesis. Literature relevant to the adhesive joint is often between various metals and resin composite as well as metal crowns and resin cement. Since PMMA and composite are both resins a brief review can lend insight to the problems facing the adhesive joint.

Poor chemical bonding can exacerbate defects mentioned earlier causing significant clinical problems, often introducing adhesive failure and increasing micro-leakage or oral fluids in the finish lines, which causes an accumulation of oral debris, microorganisms, and stains. (Bulbul \& Kesim, 2010) Many studies have concluded that chemical bonding significantly increases polymer adhesion. (Segerstrã $\llbracket[m$ \& Ruyter, 2009) Thermal cycling of the adherend and the adhesive impacts this chemical bonding. Thermal cycling is the process of cycling through two temperature extremes. This environment is often created artificially to simulate aging of dental materials in experimental design. When studying the adhesive joint between a metal and a polymer, different coefficients of thermal expansion create stresses during thermal cycling of dissimilar materials. This contraction can cause a significant decrease in shear bond strength. (Kim, Pfeiffer, \& Niedermeier, 2003) Thermal cycling can create space for moisture that can be absorbed by the polymer through hydrolysis further weakening the material. (SegerstrÃ Im \& Ruyter, 2009) The resistance of the adhesive joint to fracture is highly dependent on how all substrates react to thermo cycling. Most resin adhesion studies have demonstrated clinically that thermal cycling decreases bond strength as it weakens the interface. (Ozcan \& Kumbuloglu, 2009) A study by 
Ohkubo et al on the bonding of adhesive resin to titanium disks concluded that thermal cycling decreased the bonding of 4-META adhesive resin to commercially pure titanium. (Ohkubo, Watanabe, Hosoi, \& Okabe, 2000) Any contraction of dissimilar materials at the adhesive joint can cause strain at the interface, which stresses chemical bond strength despite external forces. (Segerstrã $\mathbb{I m} \&$ Ruyter, 2009)

\section{Airborne-Particle Abrasion}

In order to provide the best environment for chemical bonding the surface of the adherend should be prepared to receive the adhesive. One method widely used by dental laboratories and manufactures alike is airborne-particle abrasion. Airborne-particle abrasion cleans, roughens, increases the surface energy, and increases wet ability of substrates. Particles used in this process vary depending on the desired effect. The most common particles utilized in dentistry when abraiding metals is aluminum oxide. It's cheap, abundant, and can be purchased in a variety of particle sizes.

To prepare titanium, aluminum oxide or silica modified aluminum oxide can be employed. When titanium is airborne-particle abraded with silica modified aluminum oxide particles both silica particles and aluminum oxide particles are imbedded in the surface of the metal pyrolytically. Manufactures utilize this silica layer or the oxide layer of titanium to attach adhesives by chemical means. 
This surface containing silica particles can be silanated to form a stable chemical bond to resins. Silica-coating bonding systems have a long track record with removable dental prosthesis and provide a stable bond. Previous investigations have established that aluminum particles become impregnated on the surface of titanium alloys during the aluminum oxide air borne-particle abrasion process. This increases the surface content of aluminum, which can be observed with a SEM. (Ohkubo, Watanabe, Hosoi, \& Okabe, 2000)

The tribochemical silica coating technique is a well-established resin metal bonding system, and was introduced in 1989. This system requires the use of a silane-coupling agent to provide a chemical bond as well as micromechanical retention by air borne particle abrasion. (Ozcan \& Kumbuloglu, 2009) Kern used two commercial silica-coating systems to compare bond strength of Bis-GMA composite resin to titanium. Bond strength in shear increased to more than $50 \mathrm{MPa}$, which is comparable to bonding of base alloys. The specimens were subjected to 150 days of water storage yet the bond remained stable. (Kern \& Van Thompson, 1995) Susanna Segerstrom tested adhesion properties of laminated pigmented polymers with titanium. Using silanization alone also increased shear bond strength to titanium with and without airborne-particle abrasion. (SegerstrÃ Im \& Ruyter, 2009) May and colleges designed two studies which compared commercially available silica-silane bonding systems using titanium cylinders and PMMA. The first of which in 2003 concluded that the use of $110 \mu \mathrm{m}$ aluminum oxide alone had no effect on shear bond strength compared to no treatment. The addition of silane increased shear bond strength by more than 60\%. (May, Russell, Razzoog, \& Lang, 
1993) The second study incorporated $110 \mu \mathrm{m}$ silica modified aluminum oxide particles with silane bonding. Shear bond strength was increased by $68 \% \mathrm{a}$ significant difference from airborne-particle abrasion alone. (May, Fox, Razzoog, \& Lang, 1995) The bifunctional monomer in silanes is able to bond chemically to embedded alumina and/ or silica particles on the abraded metal surface. (Ozcan \& Valandro, 2011)

Literature does reflect the importance of airborne-particle abrasion to prepare the surface of metal restorations. The effect of different sized particles on the bond strength of metal resin adhesive joints is lacking. It is clear that incorporation of silane increases bond strength and the addition of silica silane bonding does as well.

\section{Primers}

Primers used in dentistry are applied to increase the adherence of one material to another. Metal alloys, before bonding, typically receive a primer coat to increase chemical linking to the polymer. These primers are applied after airborneparticle abrasion and contain molecules that bond to the metal on one end and the resin on the other. Silanes are a type of primer. Most dental primers bond composite resin to tooth structure. Metal primers perform differently when applied to different types of metal. 
Metal primers increase bond strength despite manufacturer or metal type in one study Ohkubo et al concludes, "The application of any one of the five primers tested, regardless of the brand used, significantly improved the shear bond strengths of the denture base resin to any of the cast metals tested." Metals included in this study were commercially pure titanium, Ti-6-Al-4V, and Co-Cr alloy. (Ohkubo, Watanabe, Hosoi, \& Okabe, 2000) A composite veneering study utilizing Co-Cr and titanium reported significant increase in bond strength when primed with primer. (Bulbul \& Kesim, 2010) Silane alone when applied to titanium increases adhesion of PMMA. (SegerstrÃđIm \& Ruyter, 2009)

4-methacryloxyethyl trimellitate anhydride (4-META) has been used for over 20 years to prime Co-Cr frameworks for RPD fabrication. It provides a clinically stable bond after five years of service. Jacobson's incorporation of 4-META PMMA was a novel idea; it increased overall bond strength to the surface of the Cr-Co alloy, but caused overall flexural and tensile strength weakening of PMMA. By utilizing mechanical retention, such as beads, pins, and mesh, without any chemical adhesion, surface debonding travels across a larger surface area before failure during shear testing. When crack propagation travels along this inherent surface area, clinical problems of micro leakage, water inhibition, bacterial contamination, and staining can lead to failure. When chemical bonding is incorporated, this flexure along the increased surface area interface is mitigated, resulting in an altered path of least resistance that included cohesive failure. This failure occured at a lower flexural strength in Jacobson's study. So perhaps, chemical modifiers such as 4-META are 
needed to decrease problems resulting from thermo cyclic changes but only if applied to the metal surface. (Jacobson, Chang, Keri, \& Watanabe, 1988)

Acidic functional monomers have been tested to increase the titanium resin bond with some success. Acid treatment can increase shear bond strength to titanium frameworks, however the longevity of the bond needs to be tested. (Yanagida et al., ) Application of methlylene chloride to the denture base resin during a repair can also increased shear bond strength of tested specimens. (Y. S. Sarac, Sarac, Kulunk, \& Kulunk, 2005) It is of the upmost importance that the clinician select the primer and luting material that will provide the most stable bond when construction adhesive retainers. (- Taira et al., )

Mutlu states that "there seems to be some standardization in methodology and reporting needed when testing adhesion of resin based materials onto metals." (Ozcan \& Kumbuloglu, 2009) Most studies in the literature, rely on observation of the type of adhesive joint failure. If the failure is cohesive, the sample is said to have adhered to the metal surface sufficiently to over come the stress needed to fracture PMMA resin. Grading adhesive failures in this manner is of some value, however new techniques are needed to observe what happens in real time during fracture.

\section{Opaquer}


Something that is opaque does not transmit light, is not transparent, or translucent. Titanium frameworks themselves, by definition, are opaque. During hybrid prosthesis construction, additional opaquing layers are applied to prevent absorption of light transmitted through the hybrid prosthesis. Absorption of light can create a dark silhouette indicating the location of the underlying framework. Appling pink opaquing layers can reflect color similar to the denture resin maintaining a lifelike appearance of the translucent prosthesis. Opaquer is used to obscure the color of the framework by eliminating translucent light passage. Opaquers come in many forms and are available in all of the following chemical compositions; MMA, EGDMA, UDMA, TEGDMA, bis-GMA, 4-META/MMA-TBB, and pigments. (Ozcan \& Kumbuloglu, 2009) Opaquers often contain with titanium

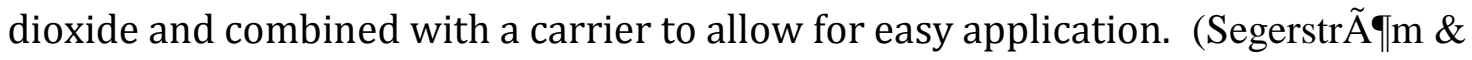
Ruyter, 2009)

Numerous previous investigations come to sound conclusions on the relationship between the metal alloy and resin as it functions as an adhesive joint. Most of these studies exclude the opaquer layer in the resin; and therefore, their conclusions have no implications clinically. Opaquers are an essential part of any framework supported prosthesis and therefore affect the adhesive joint on both chemical and mechanical fronts. Introduction of an opaquer layer in adhesive bonding often causes and adhesive type of failure. (Ozcan \& Kumbuloglu, 2009) The literature reflects lower shear bond strengths and only adhesive failures when opaque is sandwiched between the resin-metal interfaces. 
Primers that can be beneficial to metal resin bonding often contain monomers that dissolve opaquer degrading the adhesive joint. To prevent transmission of light opaque layer must have adequate thickness to perform. Thickness of opaquer can impart weakness of adhesive bonding if the opaquer is weaker than the joined substrates. One study, proposed a theory why opaquer reduces bond strength of the metal-resin joint. High polymerization contraction from additional cross-linking of the opaquer with its higher dimethacrylate concentration. (SegerstrÃ $\rrbracket$ m \& Ruyter, 2009)

Opaquer manufactures have developed few products that can join the opaquer to the titanium and the opaquer to the resin. Some products contain warnings that no chemical bond exists between opaque layer and PMMA. This joint is mechanical only and retentive elements must be created in the framework. Chemical bonding is essential to overcome the functional stresses a hybrid prosthesis undergoes in an oral enviroment.

\section{Methods and Materials}

A total of 16 identical Nobel Procera titanium alloy frameworks were manufactured to fit a master titanium block fitted with four multi-unit abutments. The frameworks were divided into five groups $(\mathrm{n}=3)$ with one sample reserved for fabrication of a metalpolyether implant fixed dental prosthesis master model. A 5mm sample of the distal extension of each bar was sectioned and set aside for SEM observation and x-ray dispersive analysis. See Figure 15. A wax-up was performed on the master block with 
the application of Heraeus Kulzer Denture teeth in an idealized set up for the size of the bar with an anterior cantilever of $10 \mathrm{~mm}$, which is commonly found clinically. See

Figures 1-4. A $1 \mathrm{~cm}$ seat was added to the lingual fosse at the midline in order to seat the universal testing machine bit. See Figure 5. A poly-vinyl siloxane (Aquasil Putty, Densply) duplication (Denture Duplicator, Lang) was made of the wax-up and indexed to the titanium framework with implant analogs torqued to $15 \mathrm{Ncm}$ in the lower flask. See Figures 6-8. Once the putty had set the wax-up was removed and the framework was steamed to remove all residual wax.

Silicone spray (Lang) was applied to both flask halves. The wax free framework was placed inside the denture duplicator flask and torqued to $15 \mathrm{Ncm}$. See figures $9-10$. A light-bodied polyether impression material (Impregum Garant Soft, 3M ESPE) was injected into all areas the mold, closed, and the compression screw was tightened. See Figure 11. Once set the metal-polyether implant fixed dental prosthesis master model was removed and four multiunit analogs were torqued to $15 \mathrm{Ncm}$ on the master model. See Figures 12-13. The metal-polyether implant fixed dental prosthesis master model with analogs in place was invested (Fast Set Laboratory Plaster, Whip Mix) and flasked (SR Ivocap System, Ivoclar Vivadent). See Figure 14. Flask halves were separated, the abutment screws were loosened, the master model was removed, and four new multi-unit abutment analogs were torqued onto the metal-polyether implant fixed complete denture master model. This technique was repeated with the same metal-polyether implant fixed complete denture master model until fifteen flasks were completed. Separating medium (Foilcote, WhipMix) was applied to each half and set aside for framework preparation. The variables between framework groups were airborne-particle abrasion and 
opaquer type in a split sample design. Test groups 1 and 2 and 3 were airborne-particle abraded with aluminum oxide particles sized 100 micron, 250 micron, and Rocatec 30 micron silica modified aluminum oxide (3M ESPE) respectively. Specimens were randomly assigned right and left halves and treated with Ropak UV opaquer (Bredent) and Telio opaquer (Ivoclar Vivadent) in split design. Micro-etcher tip held $1 \mathrm{~cm}$ from work surface at 45 -degree angle $7.2 \mathrm{bar} / 105 \mathrm{psi}$ for 5 minutes to prep each framework. See Figure 16. Handled with nylon gloves, to prevent oil contamination, frameworks were tapped on clean workbench to remove residual aluminum oxide particles. Rocatec group was treated with silane (3M ESPE). Then all specimens receiving telio were coated with SR Link (Ivoclar) application to the Telio halves. SR Link was allowed to react for 3 minutes, as recommended by manufacturer. A thin coat of Ropak opaquer (XPdent) was applied to the opposite halves. The Ropak was then cured in a UV light curing unit (XPdent) and Telio Lab opaquer was mixed with Telio Lab Opaquer liquid and applied as an even layer to all frameworks or framework halves by brush after resting for four minutes. Telio opaquer dried for 15 minutes, as recommended by manufacturer. See Figures 17-18.

Test groups four and five were treated entirely with Telio and Ropak opaquer respectively as described above. The titanium frameworks, however, were divided at the midline and randomly airborne-particle abraded with both $100 \mu$ and $250 \mu$ aluminum oxide particles.

Each of the fifteen flasks received an identical Nobel Procera titanium framework torqued to $15 \mathrm{Ncm}$ on the invested multiunit abutments. See Figure 19. Investments were flasked and processed with PMMA (SR Ivocap High Impact,) utilizing the Ivocap 
injection system, as recommended by manufacturer. Denture teeth were not incorporated to reduce processing errors. See Figure 20.

All specimens were mounted on a master titanium block fitted with replaceable multi-unit abutments. Specimens were each mounted at a 20-degree tilt to the horizon and placed in a universal testing machine at shear with a crosshead speed of $.5 \mathrm{~mm} / \mathrm{min}$ until failure. See Figures 21-22.

Tested specimens were examined with a surgical loupes (Designs for Vision) at X3.5 magnification for type of failure 1) adhesive, 2) cohesive, 3) mixed and graded as such (A, C, and M). See Table 1.

\section{SEM Surface Observation}

Of the $5 \mathrm{~mm}$ distal extension samples of each bar (32 total) 8 were picked at random and divided into 4 groups of two with one control group. Each of the four groups were prepared as follows 1) 100 micron alumina oxide 2) 250 micron alumina oxide 3) Rocatec 30 micron silica-modified alumina oxide 4) unprepared milled titanium alloy. The samples were adhered to a multiunit sample tray and the surfaces were observed with a SEM, with an accelerating voltage of $15 \mathrm{kV}$. Each sample was viewed and images were recorded at 250X, 3000X, and 27,000X. See Figure 23.

Specimens were also examined semi quantitatively with energy dispersive X-ray spectroscopy using the following settings: elevation $28.0 \mathrm{~mm}$; accelerating voltage, $15 \mathrm{kV}$; livetime, 119.9 seconds; spot size $300 \mathrm{X} 300 \mu \mathrm{m}$. Data was compiled and is depicted graphically in figure 23. Elemental compositions were obtained for each group with a randomly chosen area. Increase in surface aluminum content was noted for samples abraded with larger aluminum oxide particles. The Rocatec group had 
deposition of silica particles unlike the other samples.

\section{Load Testing}

A universal testing machine was utilized to test specimens to failure in shear with a crosshead speed of $.5 \mathrm{~mm} / \mathrm{min}$. Results were recorded graphically in Newtons/time with maximum load at failure.

\section{Results}

Maximum load at failure was recorded in table \#2 and graphically depected in Chart \#1 in Newtons. All specimens exhibited similar graphical load rates. This could indicate that the sensivity of the experimental design was not adequate to detect differences among test groups.

Oneway (ANOVA) of fit by group was performed and calculations are displayed in Table 3. Oneway Analysis of Force by group is graphically depicted in Chart 2. No statically significant difference was found among test groups. $100 \mu \mathrm{m}$ air particle abrasion group showed a slightly higher mean than the other abrasion groups. The Telio opaquer group was slightly higher than the Ropak group.

The surface area of the framework at the site of failure was consistent among all specimens $\approx 210 \mathrm{~mm}^{2}$. This area was observed with X3.5 magnification and failure type was recorded in Table 1. When Ropak was placed over $100 \mu \mathrm{m}$ air-particle abraded specimens it consistently failed with a mix of adhesion and cohesion while Telio displayed only adhesive failure. Telio presented mixed failures in combination with Rocatec $30 \mu \mathrm{m}$ silica modified aluminum oxide particles and in some $250 \mu \mathrm{m}$ surfaces. Mixed failures demonstrate increased bonding between opaquer and titanium. 


\section{Conclusion}

Within the limits of the study, the following conclusions can be drawn:

I. The air-particle abrasion techniques utilized in this study resulted in no statistically significant difference in terms of load failure.

II. The opaquer techniques utilized in this study resulted in no statically significant difference in terms of load failure.

III. With respect to type of failure there were no cohesive failures observed in this study.

IV. With respect to type of failure Ropak demonstrated mixed failure when air-particle abraded with $100 \mu \mathrm{m}$ and $250 \mu \mathrm{m}$ aluminum oxide.

V. With respect to type of failure Telio demonstrated mixed failure when air-particle abraded with Rocatec $30 \mu \mathrm{m}$ silica modified aluminum oxide. 


\section{Appendix}

Table \#1

\begin{tabular}{|c|c|c|c|c|c|}
\hline \multicolumn{6}{|c|}{ Results by Type of Failure } \\
\hline \multirow{2}{*}{$\begin{array}{l}\text { Specimen } \\
\text { A }\end{array}$} & \multirow{2}{*}{$\begin{array}{l}\text { Whole Bar } \\
100 \mu \mathrm{m}\end{array}$} & \multicolumn{2}{|c|}{ Left split } & \multicolumn{2}{|c|}{ Right split } \\
\hline & & Ropak & $\mathbf{M}$ & Telio & $\mathbf{A}$ \\
\hline B & $100 \mu \mathrm{m}$ & Telio & $\mathbf{A}$ & Ropak & M \\
\hline C & $100 \mu \mathrm{m}$ & Ropak & $\mathbf{M}$ & Telio & $\mathbf{A}$ \\
\hline D & $250 \mu \mathrm{m}$ & Telio & $\mathbf{A}$ & Ropak & $\mathbf{M}$ \\
\hline $\mathbf{E}$ & $250 \mu \mathrm{m}$ & Telio & $\mathbf{A}$ & Ropak & M \\
\hline $\mathbf{F}$ & $250 \mu \mathrm{m}$ & Ropak & $\mathbf{A}$ & Telio & $\mathbf{A}$ \\
\hline G & Rocatec & Telio & $\mathbf{M}$ & Ropak & $\mathbf{A}$ \\
\hline $\mathbf{H}$ & Rocatec & Telio & $\mathbf{M}$ & Ropak & $\mathbf{A}$ \\
\hline I & Rocatec & Ropak & $\mathbf{A}$ & Telio & M \\
\hline J & Ropak & $250 \mu \mathrm{m}$ & $\mathbf{M}$ & $100 \mu \mathrm{m}$ & M \\
\hline $\mathbf{K}$ & Ropak & $250 \mu \mathrm{m}$ & $\mathbf{A}$ & $100 \mu \mathrm{m}$ & $\mathbf{M}$ \\
\hline $\mathbf{L}$ & Ropak & $250 \mu \mathrm{m}$ & $\mathbf{A}$ & $100 \mu \mathrm{m}$ & M \\
\hline $\mathbf{M}$ & Telio & $100 \mu \mathrm{m}$ & $\mathbf{A}$ & $250 \mu \mathrm{m}$ & $\mathbf{M}$ \\
\hline $\mathbf{N}$ & Telio & $250 \mu \mathrm{m}$ & $\mathbf{M}$ & $100 \mu \mathrm{m}$ & $\mathbf{A}$ \\
\hline $\mathbf{0}$ & Telio & $250 \mu \mathrm{m}$ & $\mathbf{M}$ & $100 \mu \mathrm{m}$ & $\mathbf{A}$ \\
\hline \multicolumn{6}{|c|}{$\begin{array}{l}\text { A = Adhesive Failure } \\
\text { M = Mixed Failure } \\
\text { There Were No Cohesive Failures In This Study }\end{array}$} \\
\hline
\end{tabular}


Table \#2

\begin{tabular}{|c|c|}
\hline \multicolumn{2}{|c|}{ Results by Load Failure } \\
\hline Specimen & Force (Newtons) \\
\hline A & 3362.9 \\
\hline B & 3490.0 \\
\hline C & 3632.9 \\
\hline D & 3055.0 \\
\hline E & 3384.3 \\
\hline F & 3164.6 \\
\hline G & 3250.1 \\
\hline H & 3503.3 \\
\hline I & 3262.0 \\
\hline K & 3194.9 \\
\hline L & 3238.7 \\
\hline N & 3660.4 \\
\hline O & 3257.7 \\
\hline
\end{tabular}




\section{Table \#3 Group}

\section{Oneway Anova \\ Summary of Fit}

Rsquare

Adj Rsquare

Root Mean Square Error

Mean of Response

Observations (or Sum Wgts)
0.289458

0.005241

190.3972

3366.753

\section{Analysis of Variance}

$\begin{array}{lrrrrr}\text { Source } & \text { DF } & \begin{array}{r}\text { Sum of } \\ \text { Squares }\end{array} & \text { Mean Square } & \text { F Ratio } & \text { Prob > F } \\ \text { Group } & 4 & 147678.17 & 36919.5 & 1.0184 & 0.4432 \\ \text { Error } & 10 & 362510.81 & 36251.1 & & \\ \text { C. Total } & 14 & 510188.98 & & & \end{array}$

\section{Means for Oneway Anova}

$\begin{array}{lrrrrr}\text { Level } & \text { Number } & \text { Mean } & \text { Std Error } & \text { Lower 95\% } & \text { Upper 95\% } \\ 100 & 3 & 3495.27 & 109.93 & 3250.3 & 3740.2 \\ 250 & 3 & 3201.30 & 109.93 & 2956.4 & 3446.2 \\ \text { Rocatec } & 3 & 3338.47 & 109.93 & 3093.5 & 3583.4 \\ \text { Ropak } & 3 & 3364.67 & 109.93 & 3119.7 & 3609.6 \\ \text { Telio } & 3 & 3434.07 & 109.93 & 3189.1 & 3679.0\end{array}$

Std Error uses a pooled estimate of error variance

\begin{tabular}{|c|c|c|c|c|c|}
\hline \multicolumn{6}{|c|}{ Van der Waerden Test (Normal Quantiles) } \\
\hline Level & Count & Score Sum & $\begin{array}{r}\text { Expected } \\
\text { Score }\end{array}$ & Score Mean & (Mean-Mean0)/Std0 \\
\hline 100 & 3 & 1.376 & 0.000 & 0.45864 & 1.022 \\
\hline 250 & 3 & -2.366 & 0.000 & -0.78861 & -1.758 \\
\hline Rocatec & 3 & 0.028 & 0.000 & 0.00947 & 0.021 \\
\hline Ropak & 3 & -0.411 & 0.000 & -0.13710 & -0.306 \\
\hline Telio & 3 & 1.373 & 0.000 & 0.45760 & 1.020 \\
\hline
\end{tabular}

1-way Test, ChiSquare Approximation

ChiSquare DF Prob>ChiSq

$\begin{array}{lll}4.2149 & 4 & 0.3777\end{array}$




\section{Chart \#1}

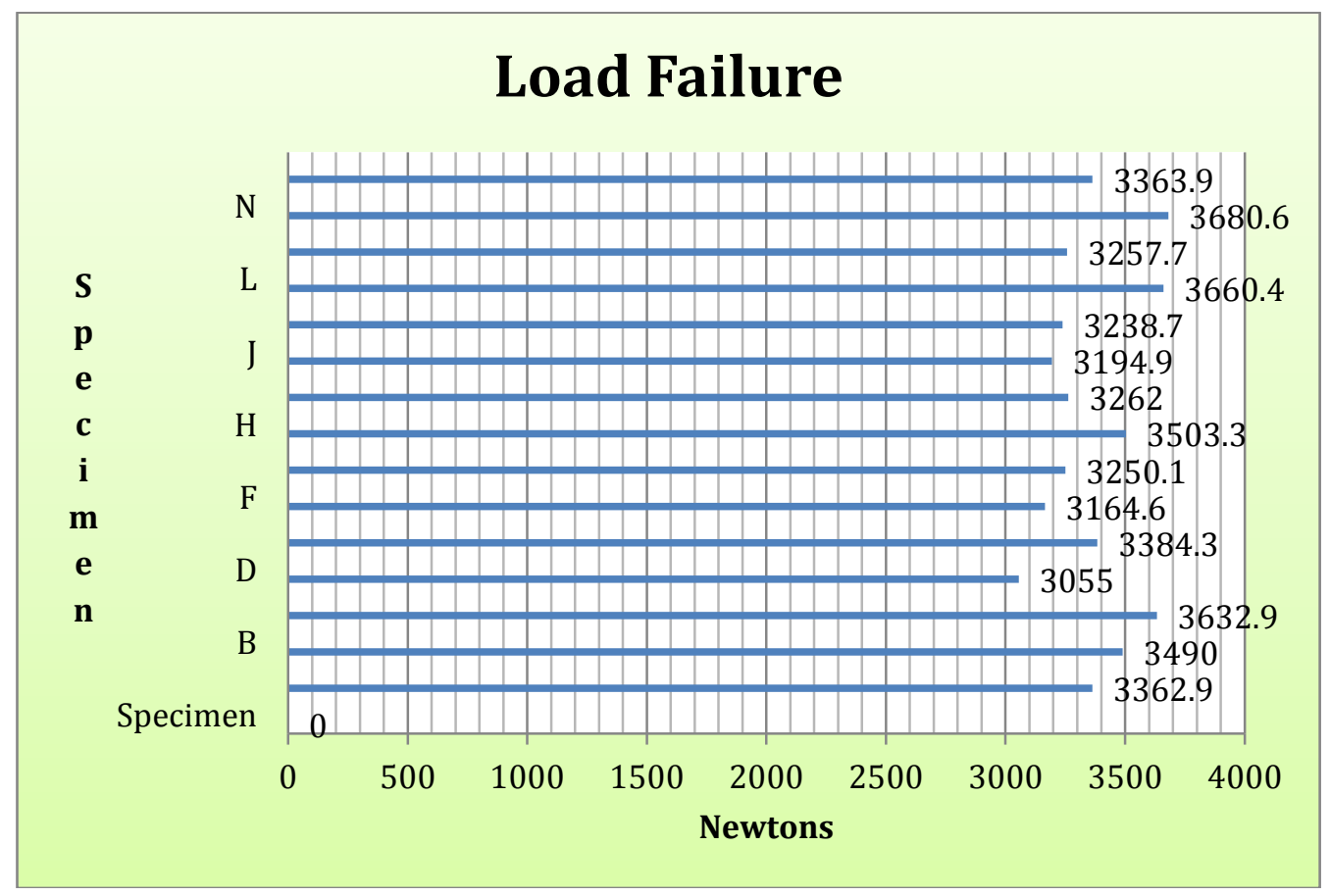

\section{Chart \#2}

Oneway Analysis of Force By Group

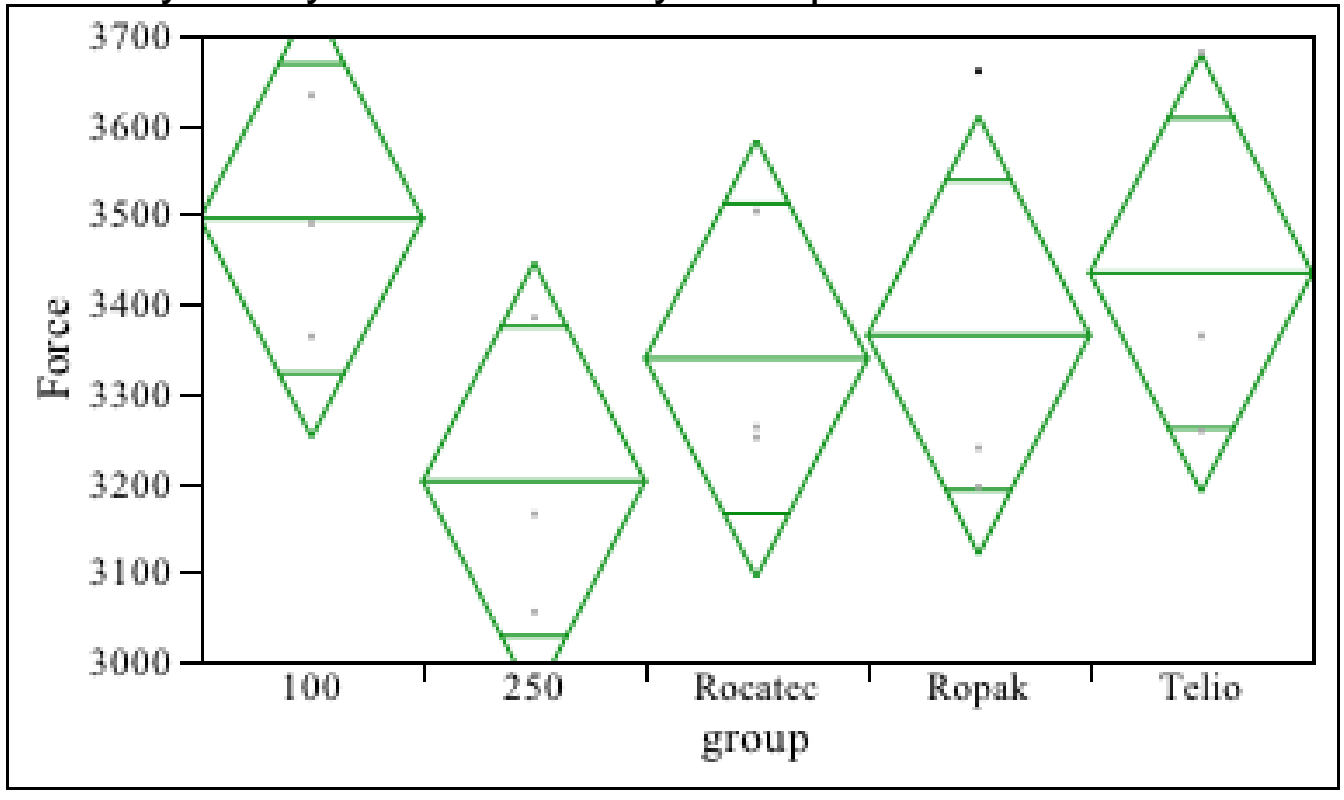




\section{Images}

Figure 1

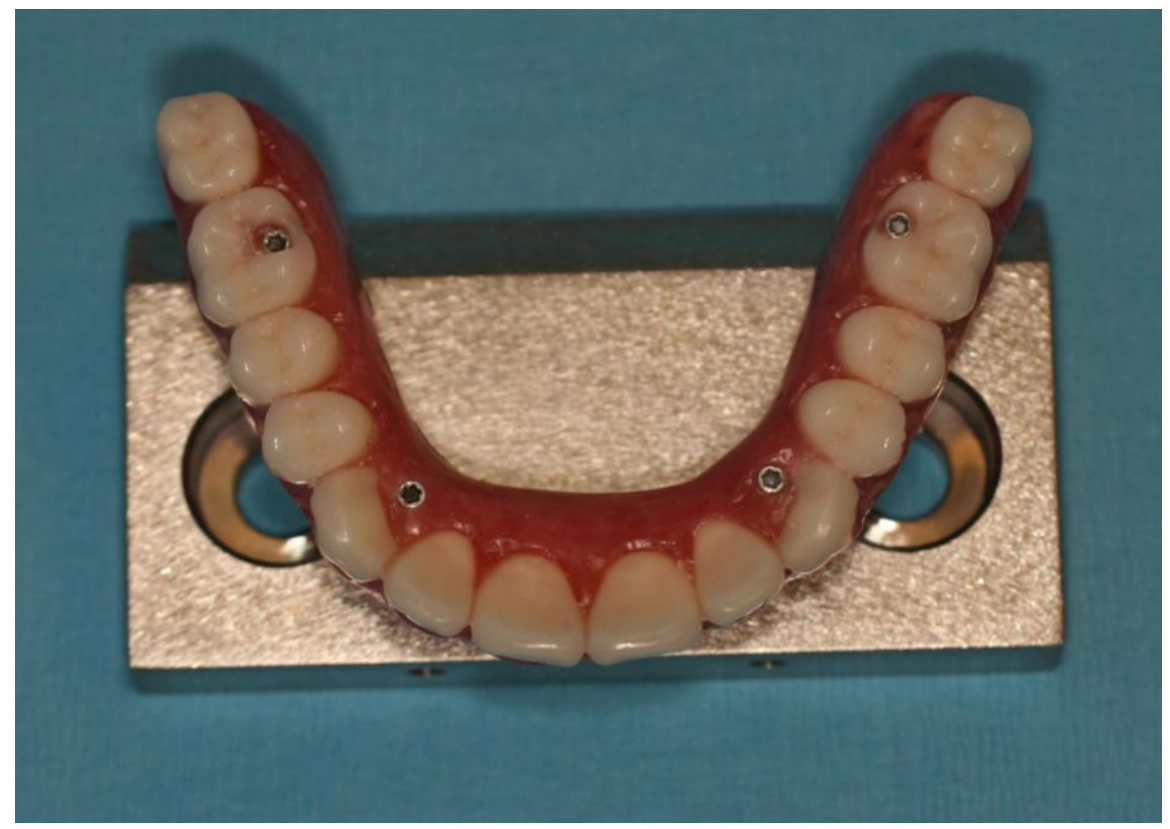

Figure 2

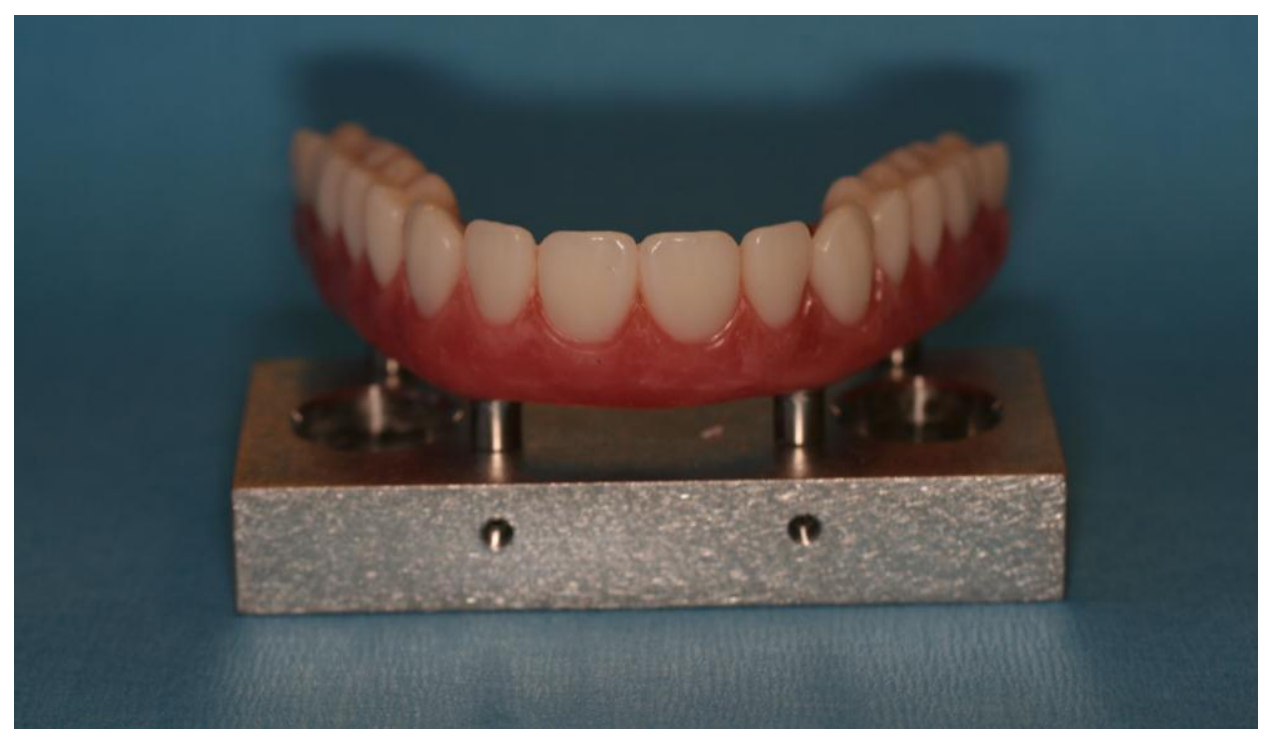


Figure 3

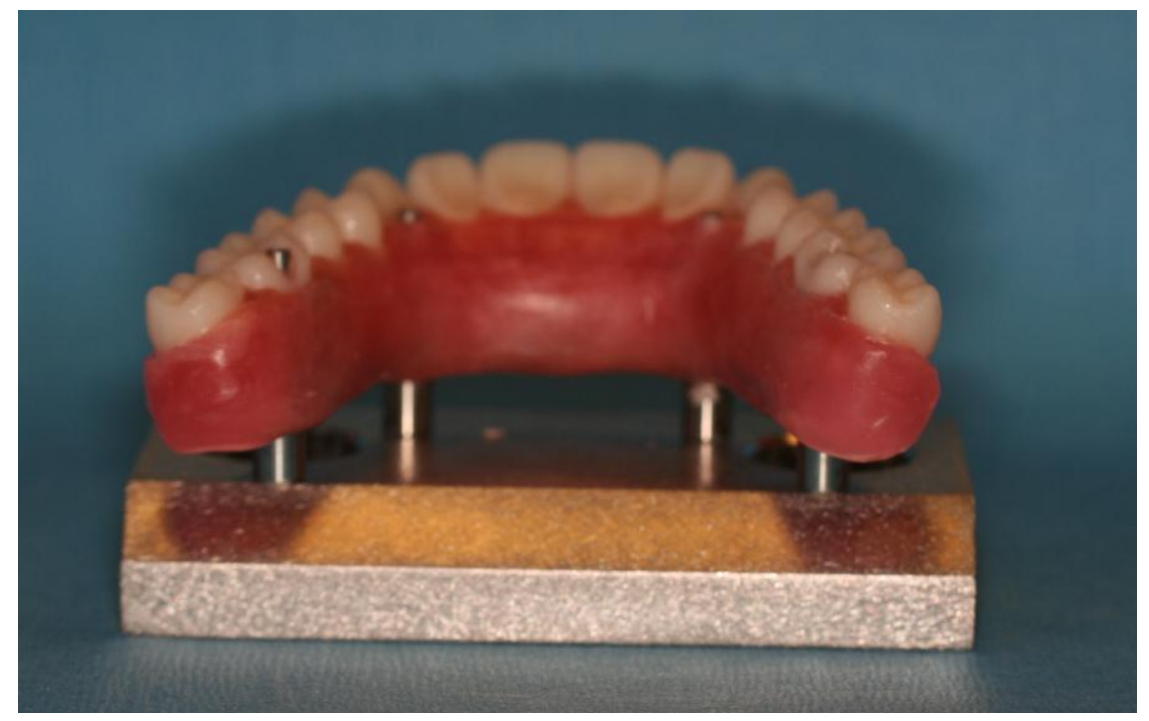

Figure 4

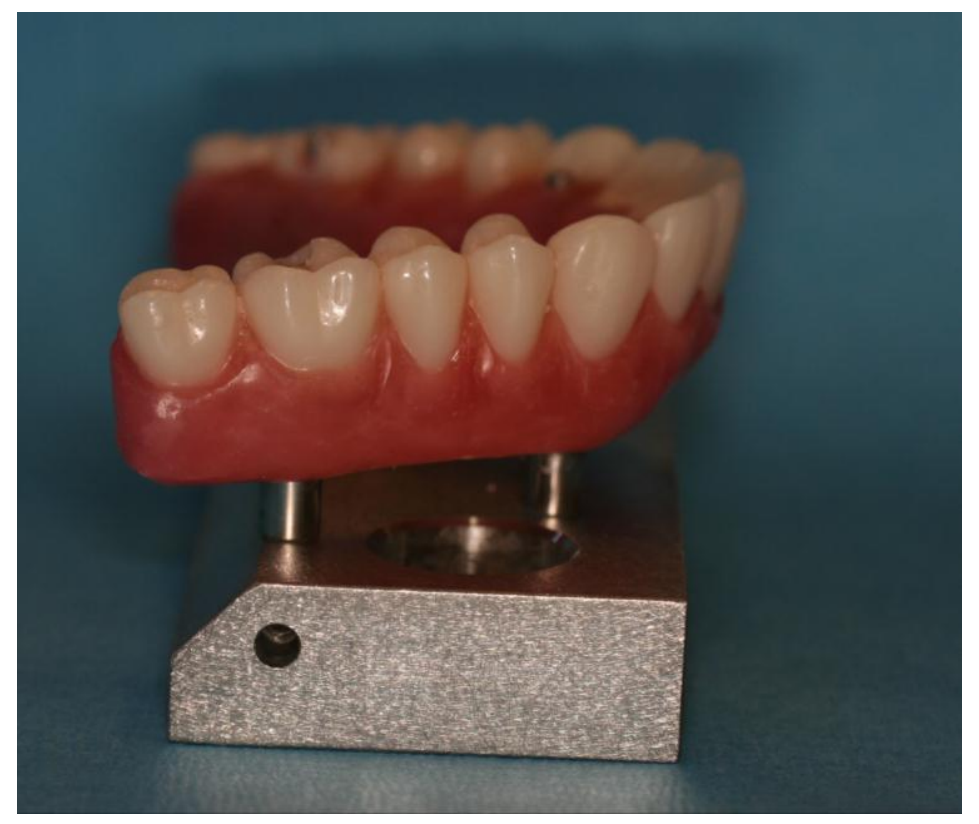


Figure 5

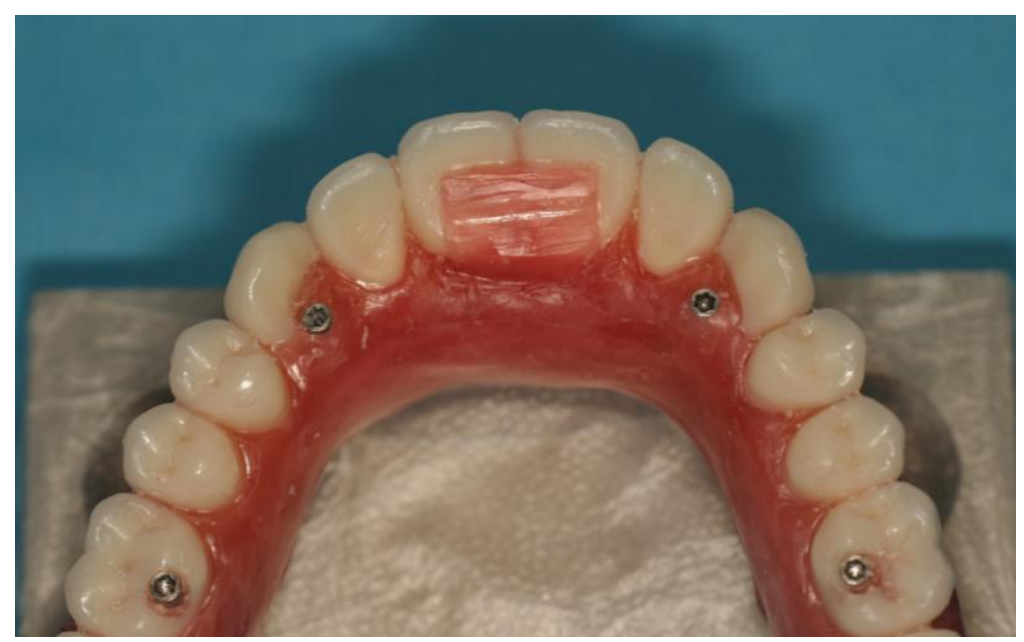

Figure 6

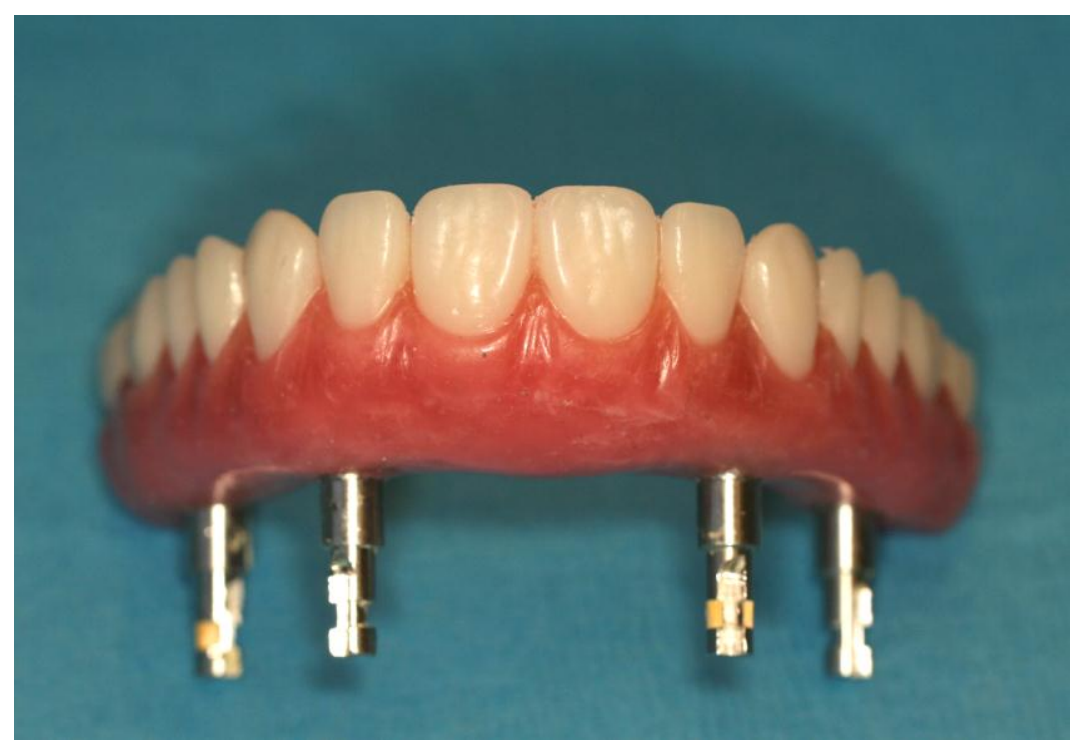


Figure 7

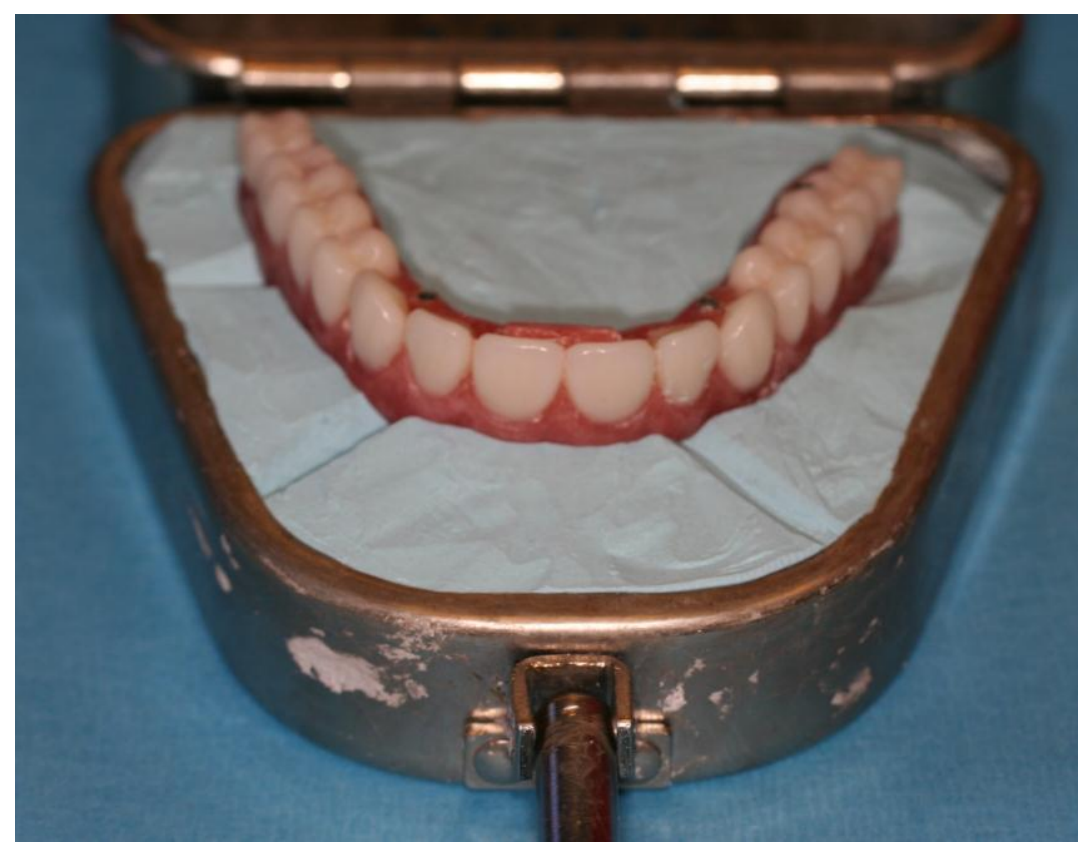

Figure 8

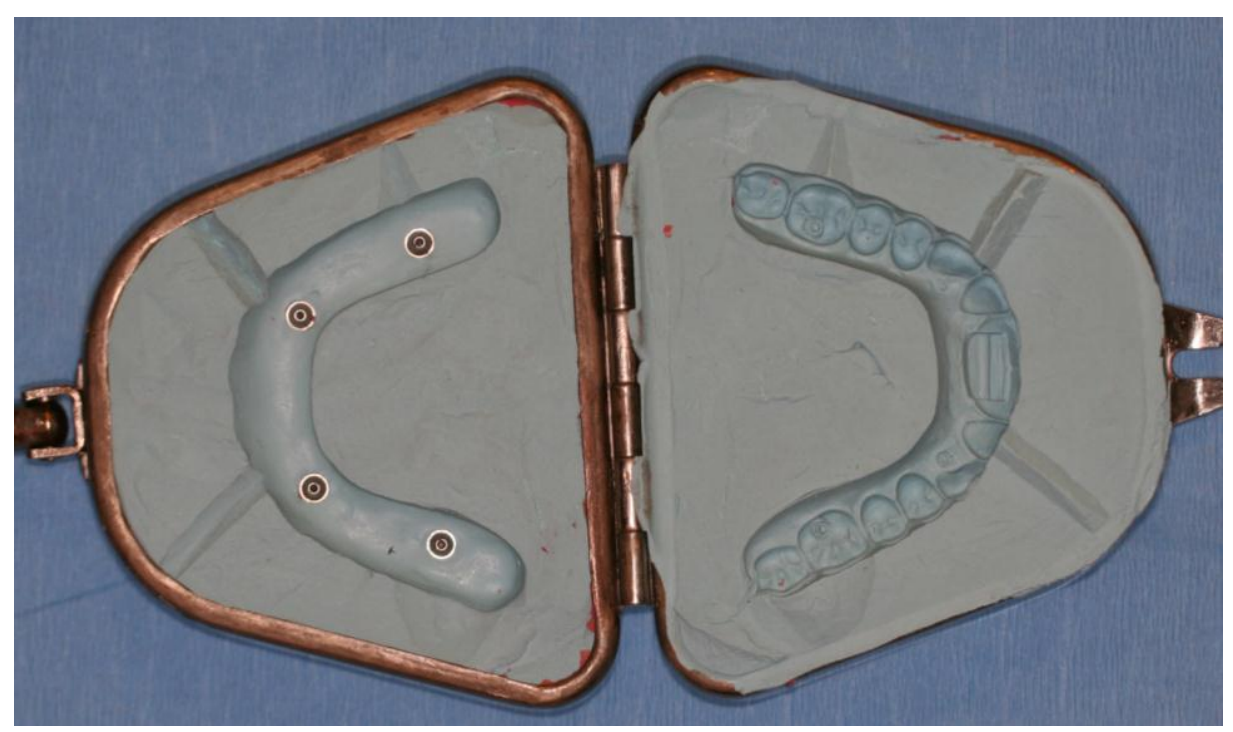


Figure 9

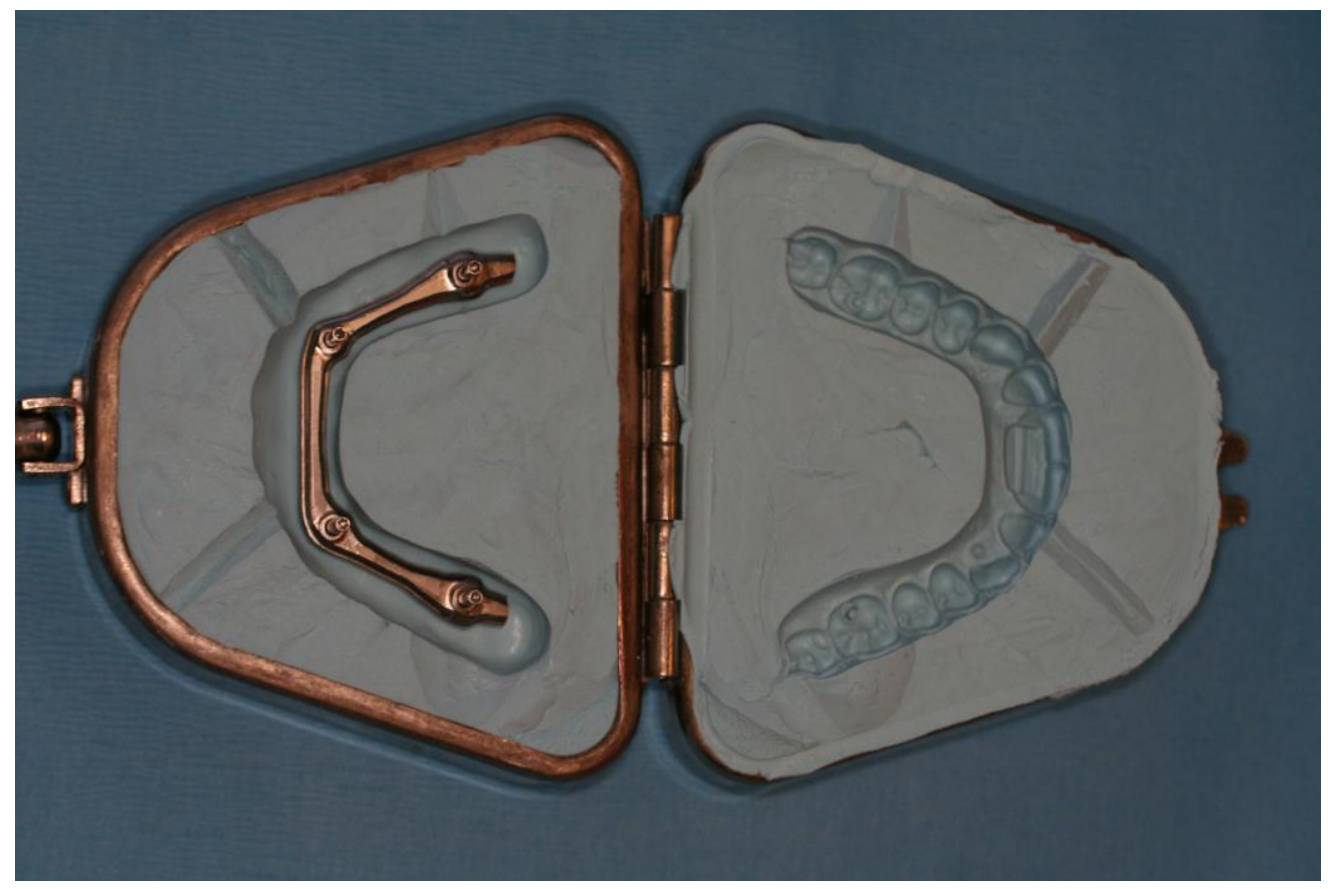

Figure 10

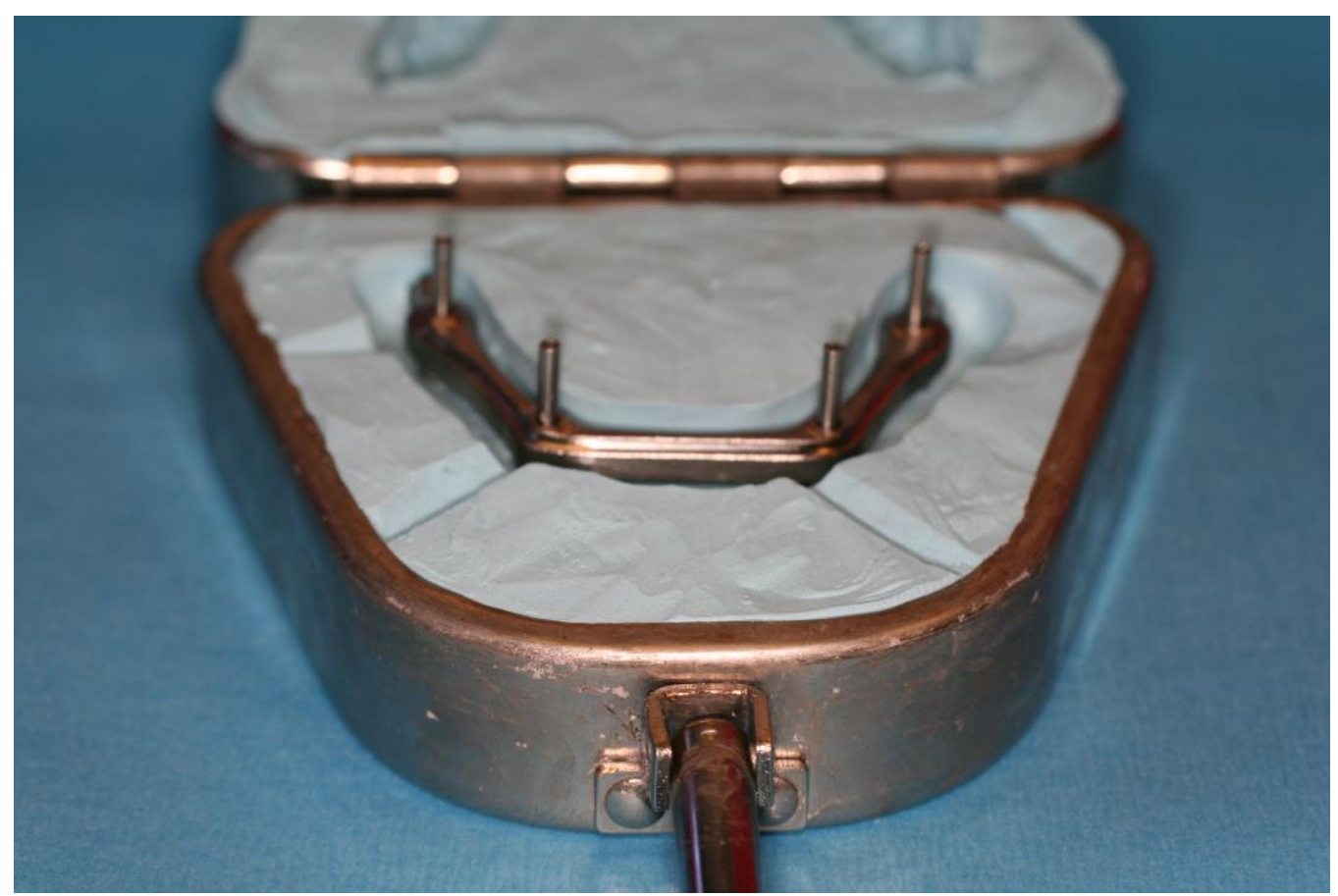


30

Figure 11

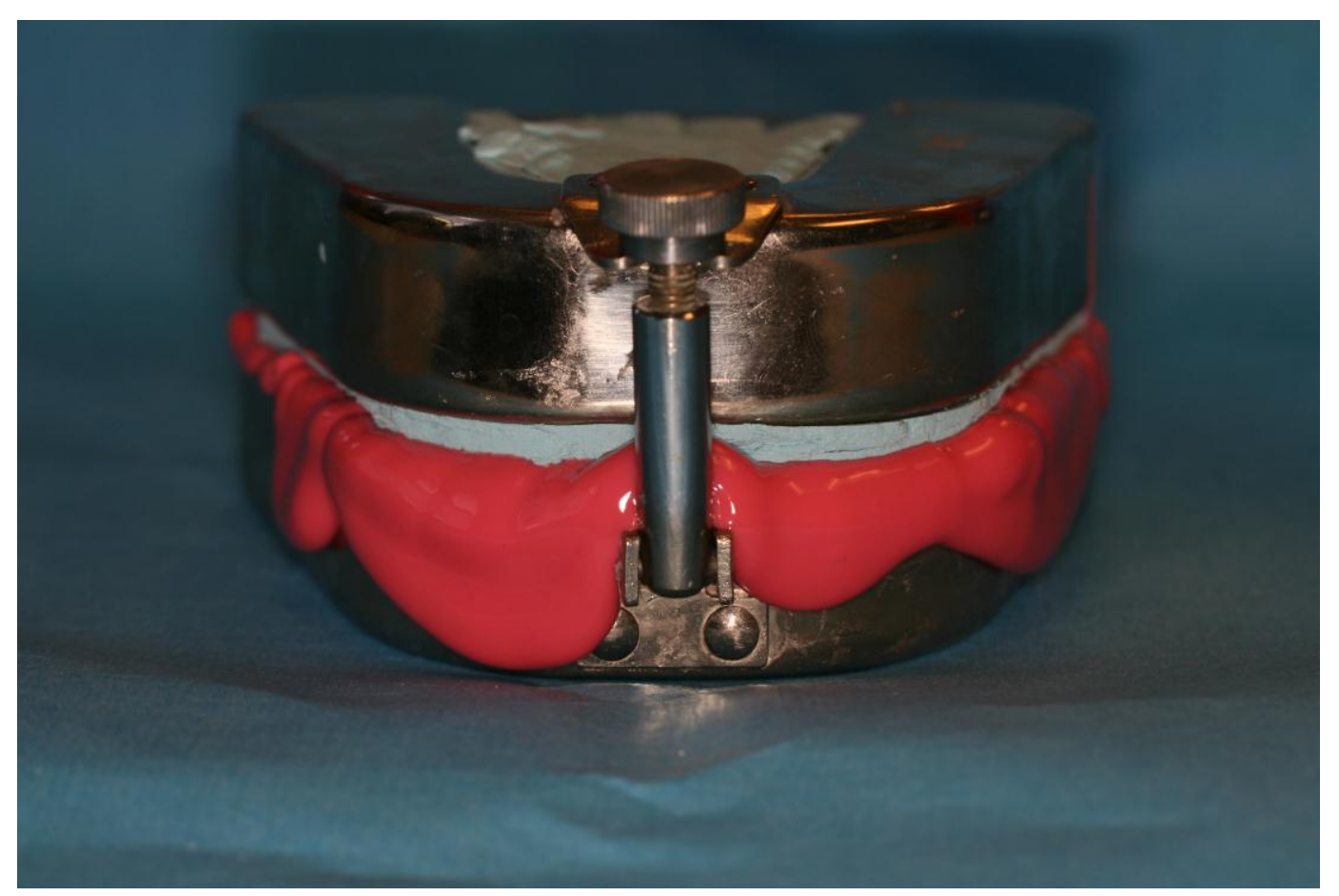

Figure 12 
Figure 13
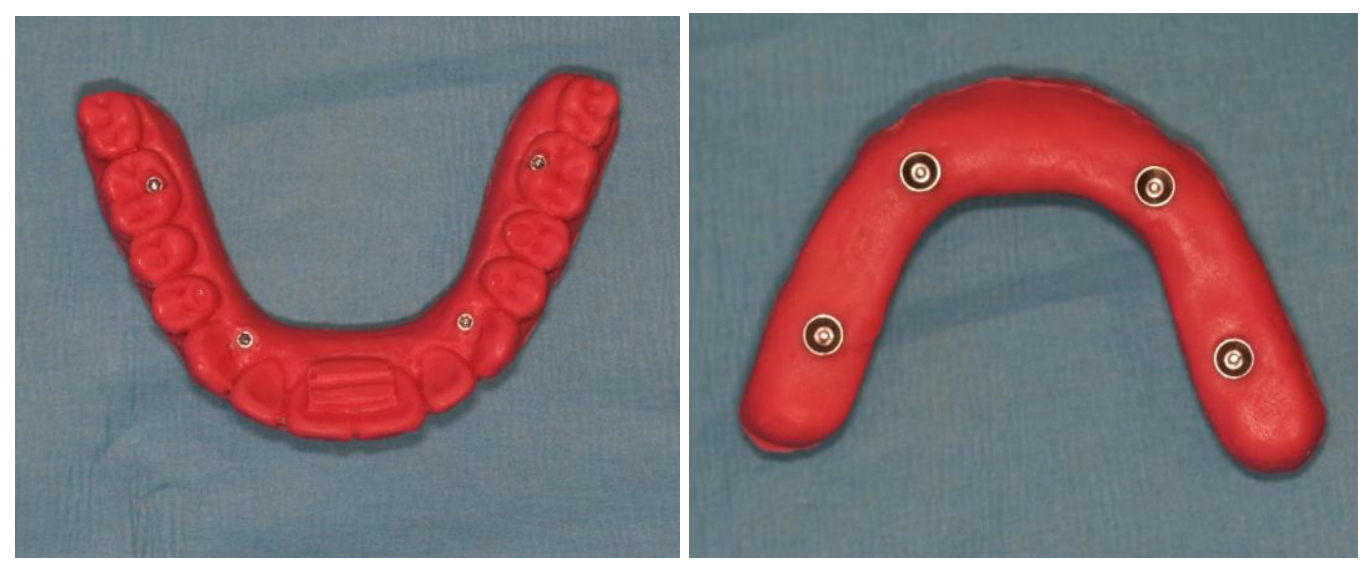

Figure 14

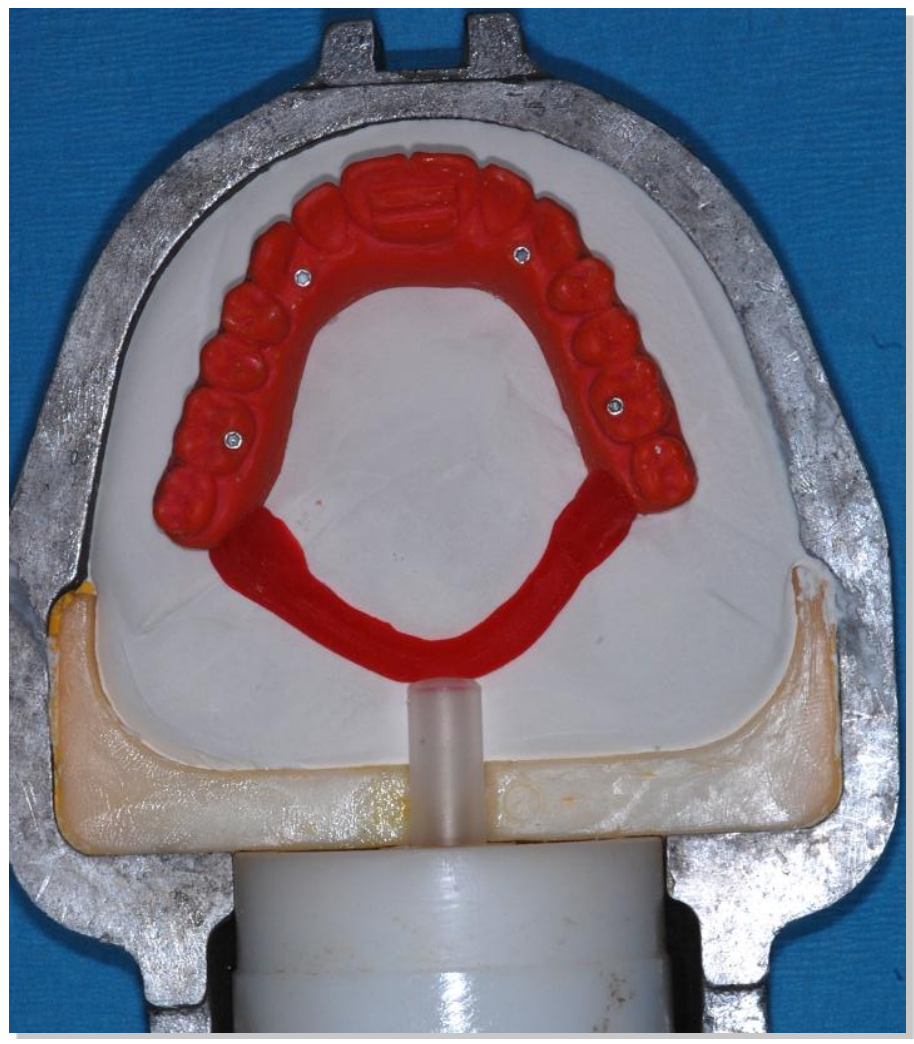


Figure 15

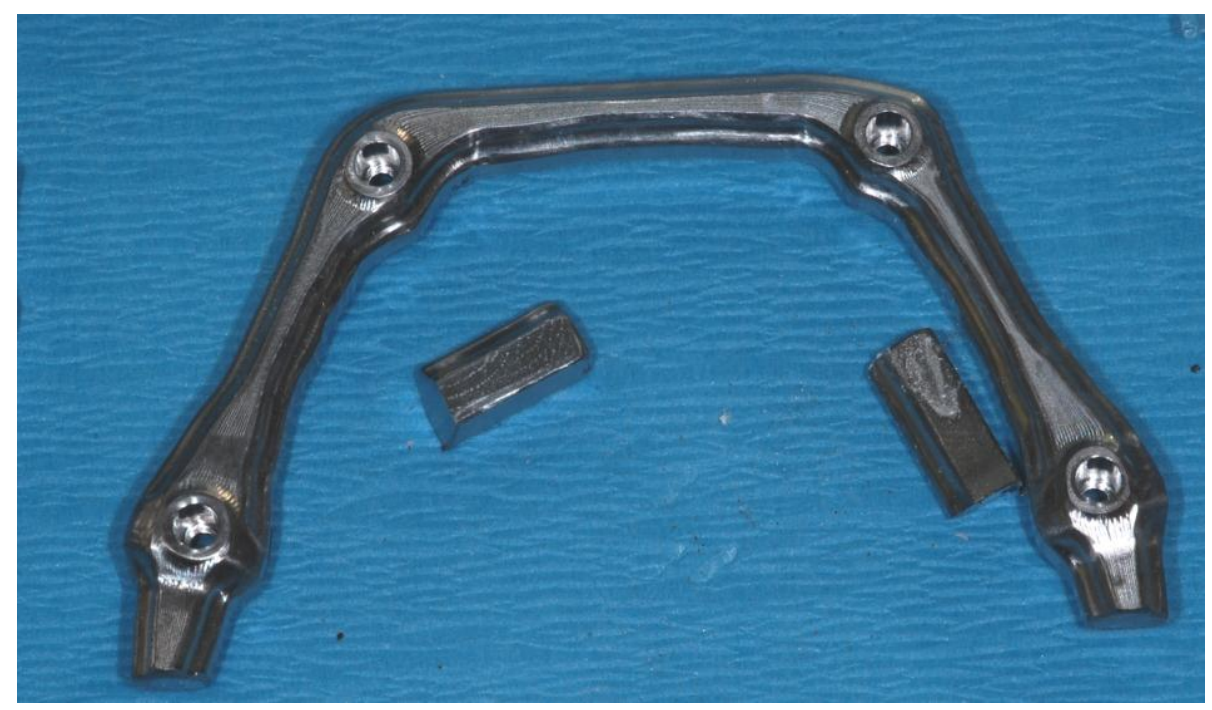

Figure 16

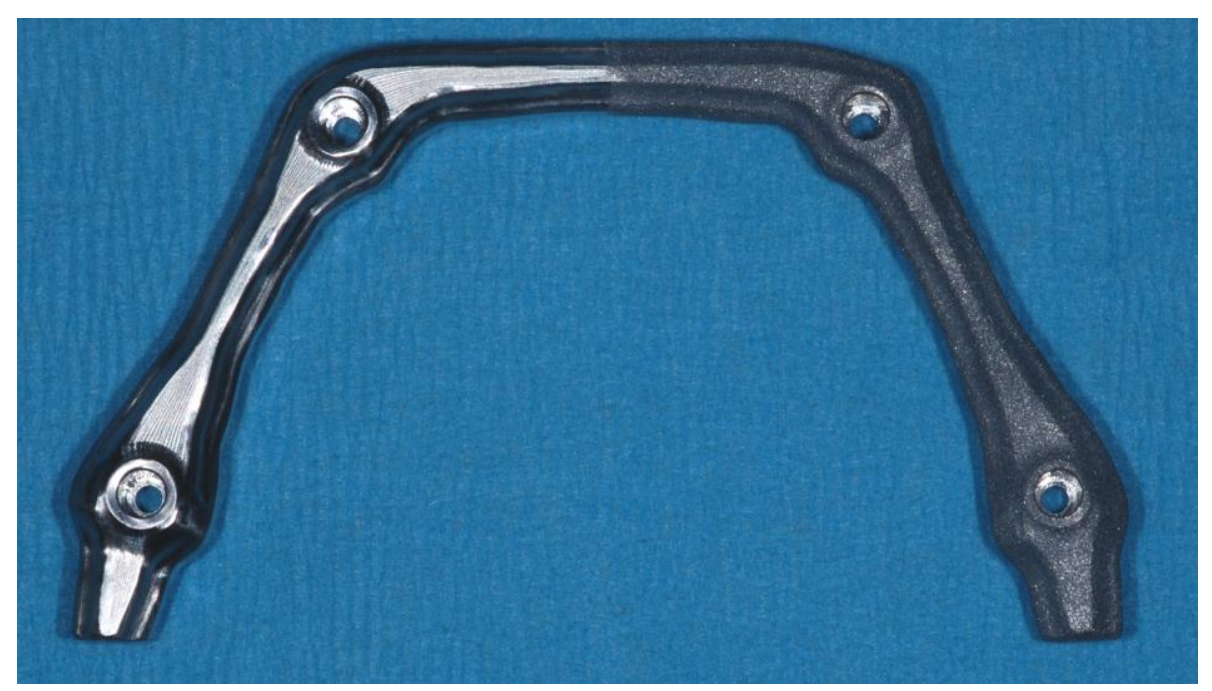


Figure 17

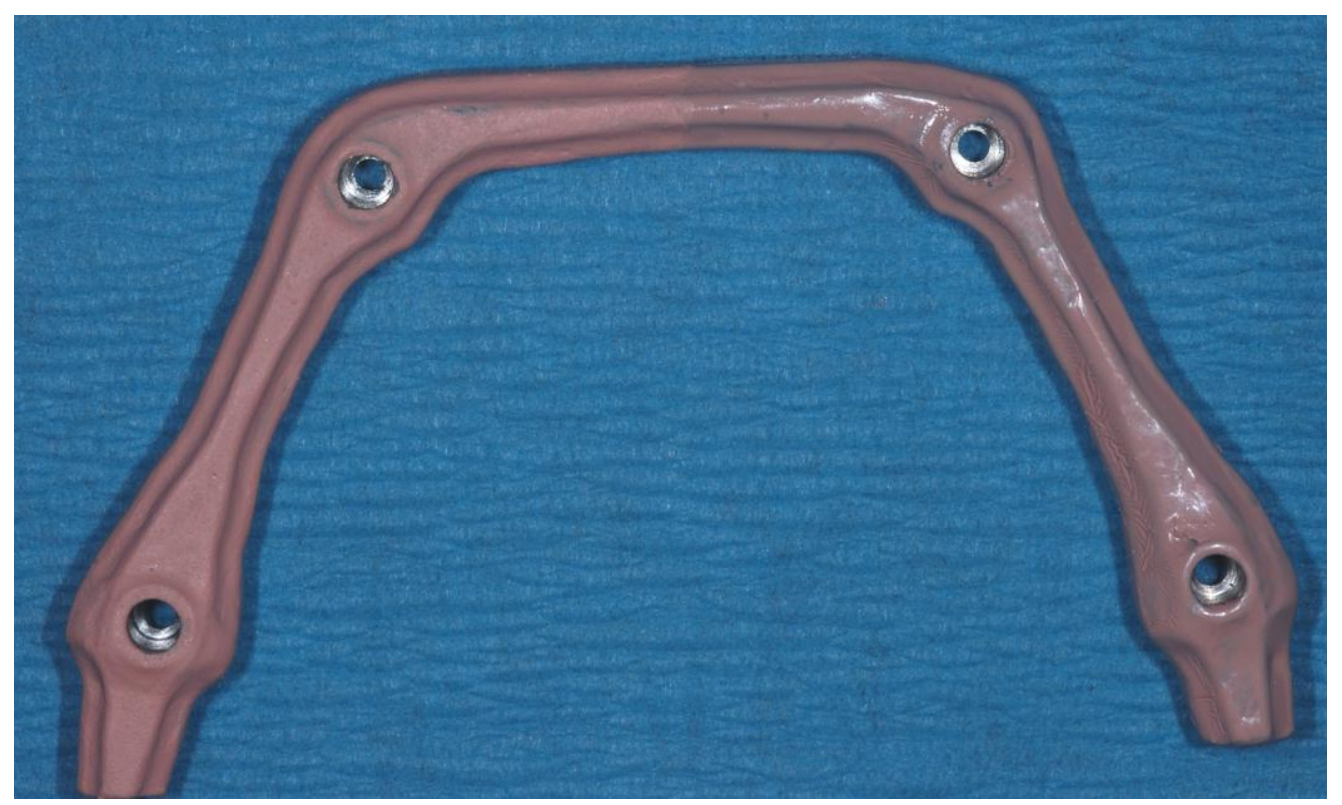

Figure 18

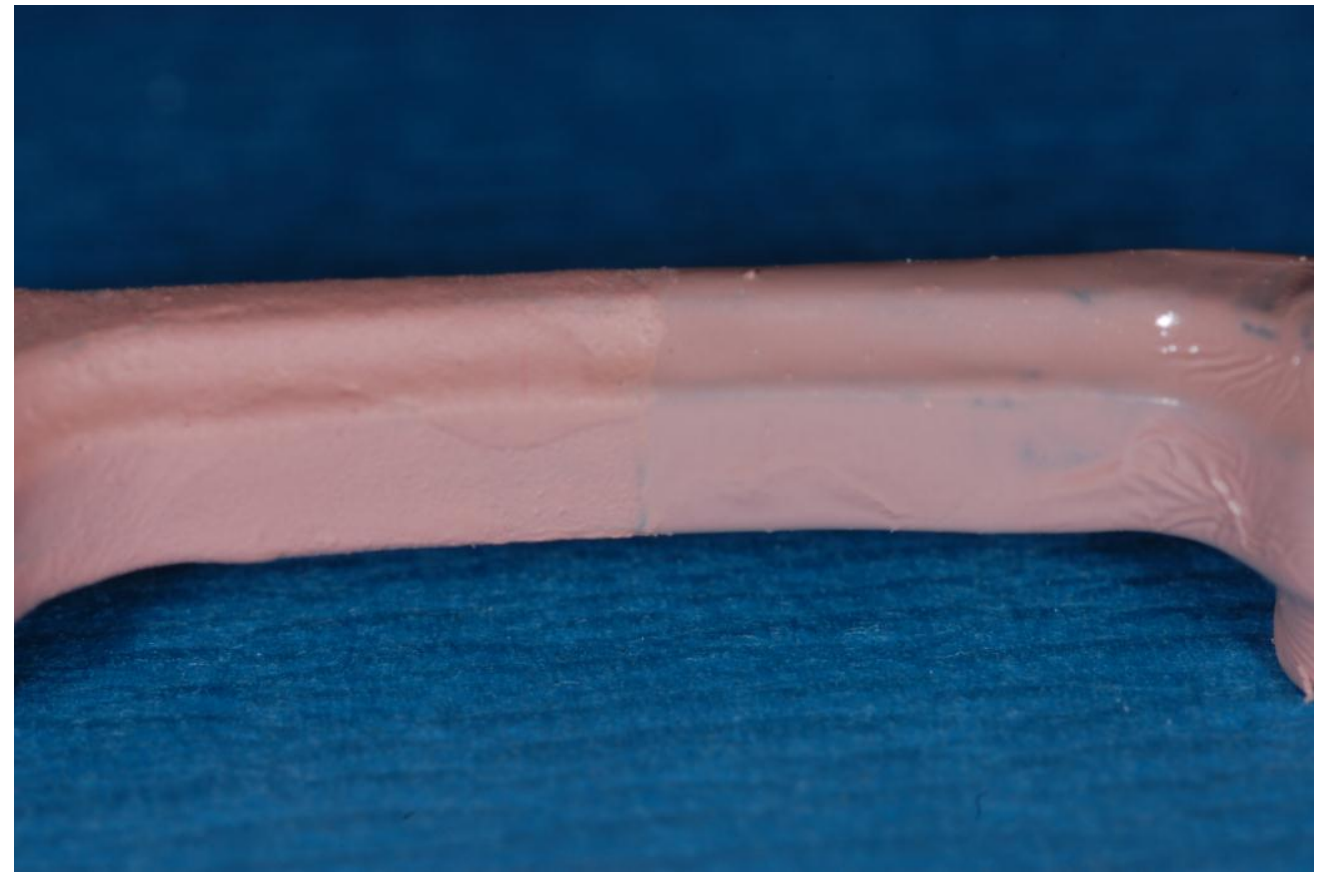


Figure 19

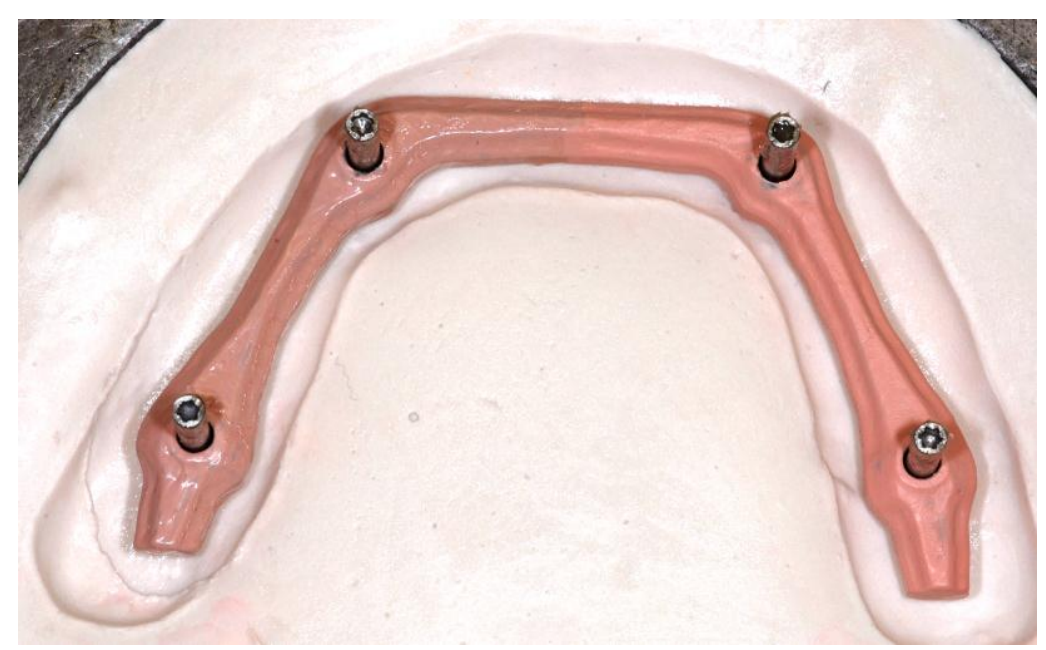

Figure 20
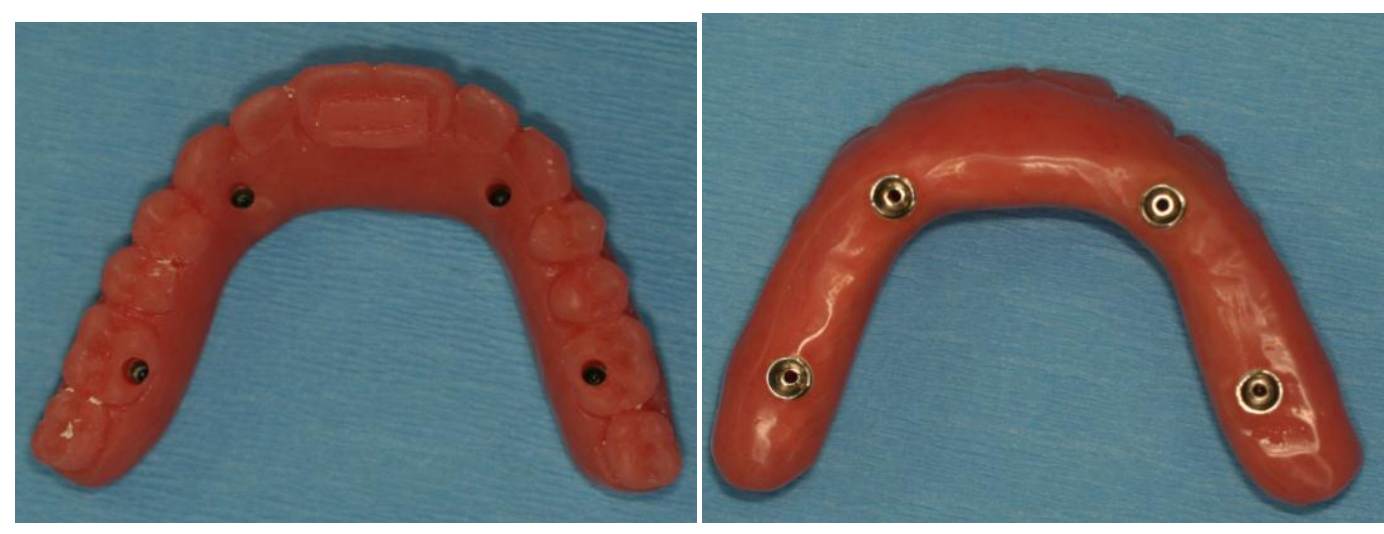
Figure 21

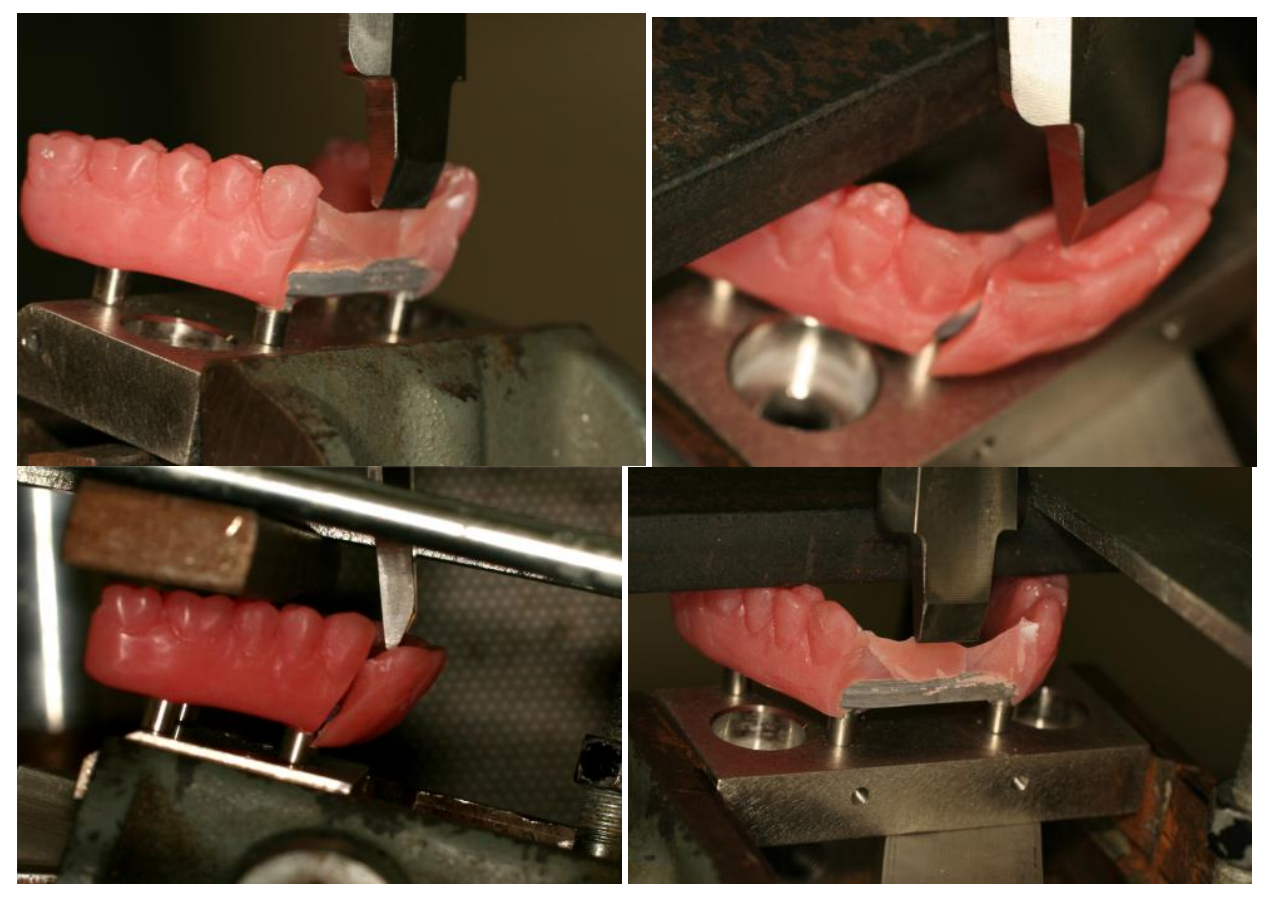

Figure 22

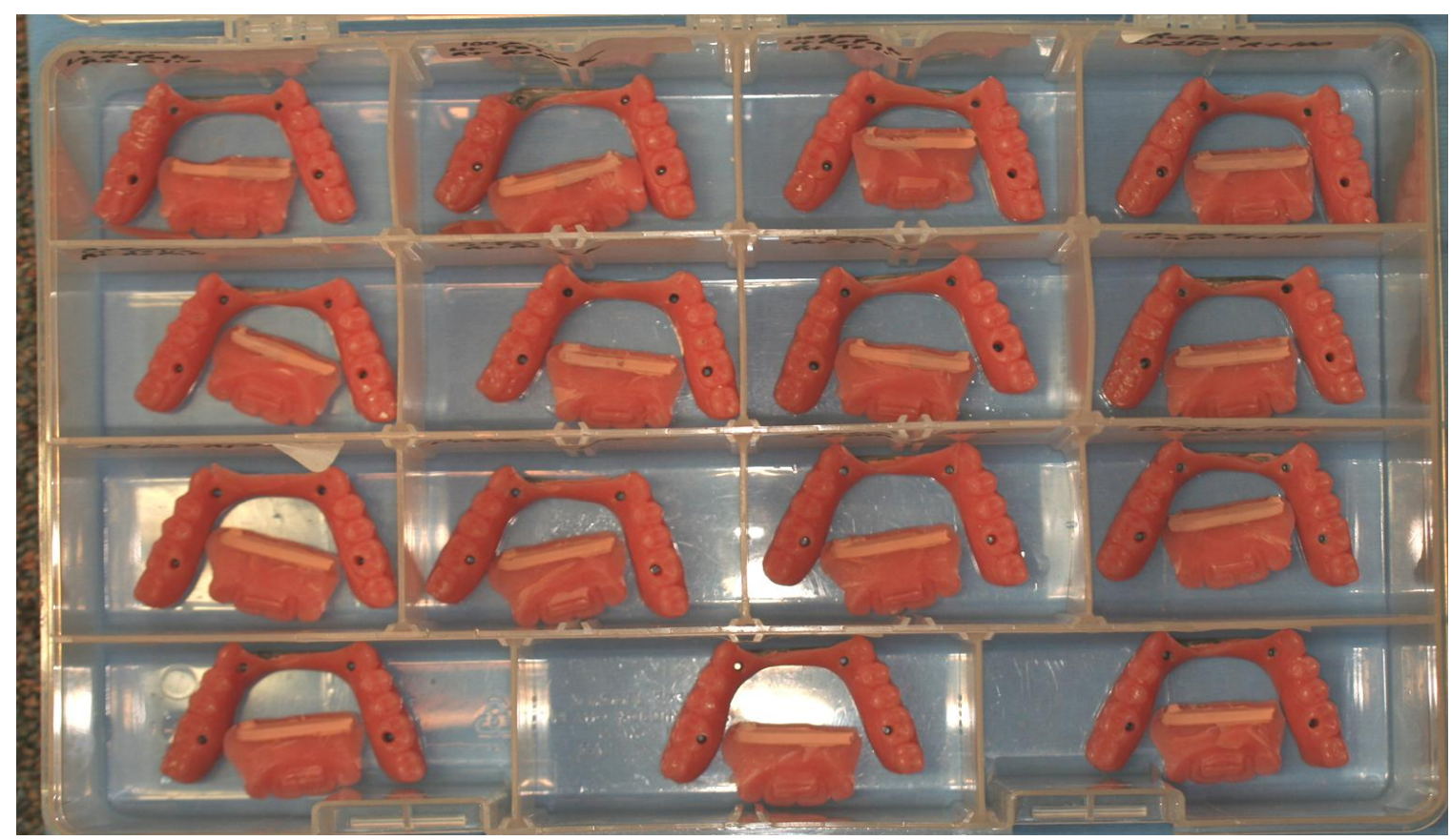


Figure 23

Energy-Dispersive X-ray Spectroscopy

SEM Figures
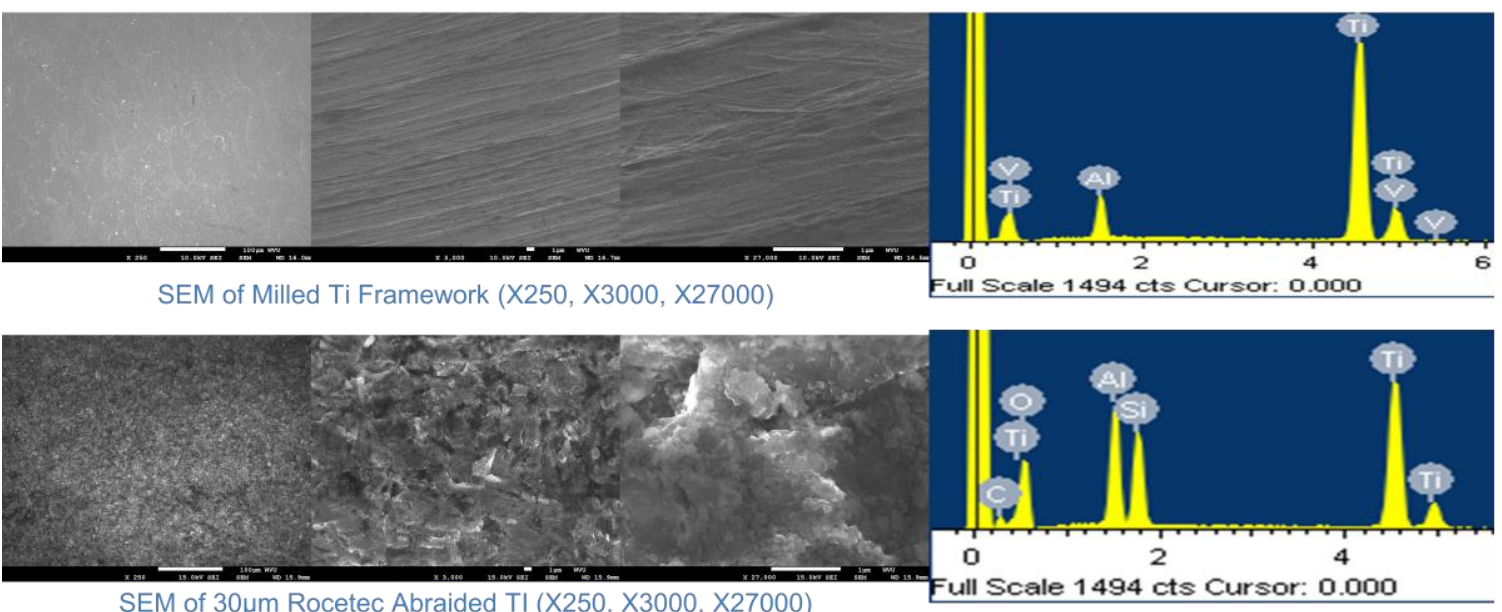

SEM of 30um Rocetec Abraided TI (X250, X3000, X27000)

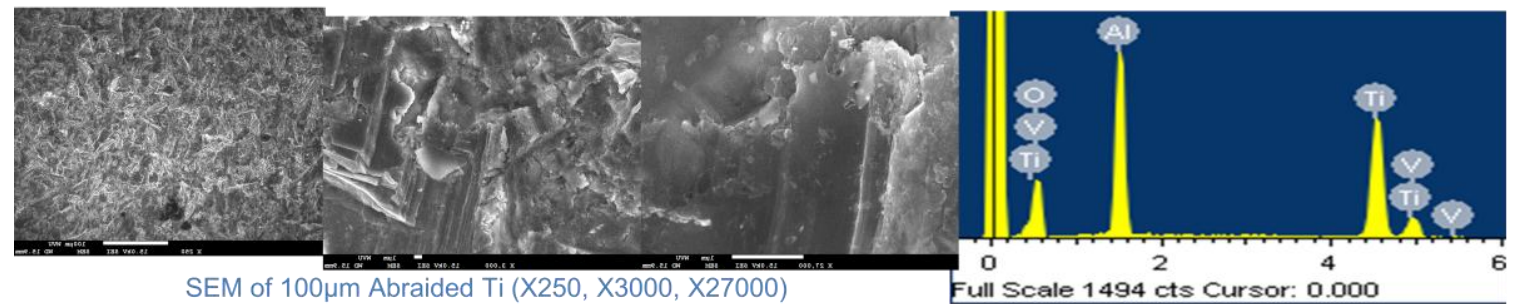

SEM of $100 \mu \mathrm{m}$ Abraided Ti (X250, X3000, X27000)

Full Scale 1494 cts Cursor: 0.000

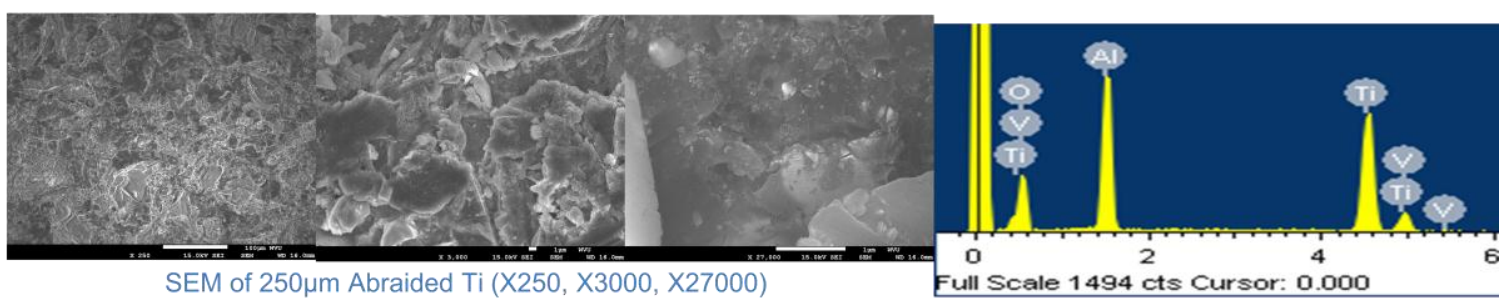




\section{References}

- Örtorp, A., \& - Jemt, T. (a). - Clinical experience of CNC-milled titanium frameworks supported by implants in the edentulous jaw: A 3-year interim report - Blackwell Publishing Ltd.

- Örtorp, A., \& - Jemt, T. (b). - Clinical experiences of computer numeric control-milled titanium frameworks supported by implants in the edentulous jaw: A 5-year prospective study - Blackwell Publishing Ltd.

- Taira, Y., - Yanagida, H., - Matsumura, H., - Yoshida, K., - Atsuta, M., \& - Suzuki, S. Adhesive bonding of titanium with a thione-phosphate dual functional primer and self-curing luting agents - Munksgaard International Publishers.

- Yanagida, H., - Taira, Y., - Shimoe, S., - Atsuta, M., - Yoneyama, T., \& - Matsumura, H. - Adhesive bonding of titanium-aluminum-niobium alloy with nine surface preparations and three self-curing resins - Munksgaard International Publishers.

Al-Fadda, S. A., Zarb, G. A., \& Finer, Y. (2007). A comparison of the accuracy of fit of 2 methods for fabricating implant-prosthodontic frameworks. The International Journal of Prosthodontics, 20(2), 125-131.

Bulbul, M., \& Kesim, B. (2010). The effect of primers on shear bond strength of acrylic resins to different types of metals. The Journal of Prosthetic Dentistry, 103(5), 303308. 
Burns, D. R., Beck, D. A., \& Nelson, S. K. (2003). A review of selected dental literature on contemporary provisional fixed prosthodontic treatment: Report of the committee on research in fixed prosthodontics of the academy of fixed prosthodontics. The Journal of Prosthetic Dentistry, 90(5), 474-497. doi:10.1016/S0022-3913(03)002592

Craig, R. G., \& Powers, J. M. In Craig R. G., Powers J. M. (Eds.), Restorative dental materials (11th ed.). St. Louis, Missouri: Mosby.

Engstrand, P., Nannmark, U., Martensson, L., Galeus, I., \& Branemark, P. I. (2001). Branemark novum: Prosthodontic and dental laboratory procedures for fabrication of a fixed prosthesis on the day of surgery. The International Journal of Prosthodontics, 14(4), 303-309.

Fujishima, A. A. (1995). Shear bond strength of four commercial bonding systems to cpTi. Dental Materials, 11(2), 82-86.

Gladwin, M. A., \& Bagby, M. D. (2009). Clinical aspects of dental materials :Theory, practice, and cases /marcia gladwin, michael bagby. Philadelphia: Wolters Kluwer Health/Lippincott Williams \& Wilkins.

Jacobson, T. E., Chang, J. C., Keri, P. P., \& Watanabe, L. G. (1988). Bond strength of 4META acrylic resin denture base to cobalt chromium alloy. The Journal of Prosthetic Dentistry, 60(5), 570-576. 
Kern, M., \& Van Thompson, P. (1995). Durability of resin bonds to pure titanium. Journal of Prosthodontics, 4(1), 16-22. doi:10.1111/j.1532-849X.1995.tb00309.x

Kim, J., Pfeiffer, P., \& Niedermeier, W. (2003). Effect of laboratory procedures and thermocycling on the shear bond strength of resin-metal bonding systems. The Journal of Prosthetic Dentistry, 90(2), 184-189.

May, K. B., Russell, M. M., Razzoog, M. E., \& Lang, B. R. (1993). The shear strength of polymethyl methacrylate bonded to titanium partial denture framework material. The Journal of Prosthetic Dentistry, 70(5), 410-413.

May, K. B., Fox, J., Razzoog, M. E., \& Lang, B. R. (1995). Silane to enhance the bond between polymethyl methacrylate and titanium. The Journal of Prosthetic Dentistry, 73(5), 428-431.

Ohkubo, C., Watanabe, I., Hosoi, T., \& Okabe, T. (2000). Shear bond strengths of polymethyl methacrylate to cast titanium and cobalt-chromium frameworks using five metal primers. The Journal of Prosthetic Dentistry, 83(1), 50-57. doi:10.1016/S0022-3913(00)70088-6

Ortorp, A., Jemt, T., Back, T., \& Jalevik, T. (2003). Comparisons of precision of fit between cast and CNC-milled titanium implant frameworks for the edentulous mandible. The International Journal of Prosthodontics, 16(2), 194-200.

Ozcan, M., \& Kumbuloglu, O. (2009). Effect of composition, viscosity and thickness of the opaquer on the adhesion of resin composite to titanium. Dental Materials : 
Official Publication of the Academy of Dental Materials, 25(10), 1248-1255. doi:10.1016/j.dental.2009.05.005

Parel, S. S. M. (2011). A risk assessment treatment planning protocol for the four implant immediately loaded maxilla: Preliminary findings. The Journal of Prosthetic Dentistry, 106(6), 359-366.

Sarac, Y. S., Sarac, D., Kulunk, T., \& Kulunk, S. (2005). The effect of chemical surface treatments of different denture base resins on the shear bond strength of denture repair. The Journal of Prosthetic Dentistry, 94(3), 259-266.

SegerstrÃ Im, S., \& Ruyter, I. E. (2009). Adhesion properties in systems of laminated pigmented polymers, carbonâ€"graphite fiber composite framework and titanium surfaces in implant suprastructures. Dental Materials, 25(9), 1169-1177.

Shackleton, J. L. (1994). Survival of fixed implant-supported prostheses related to cantilever lengths. The Journal of Prosthetic Dentistry, 71(1), 23. 